\title{
A Bayesian Account of Memory for Text
}

\author{
Mark Andrews ${ }^{1}$ \\ ${ }^{1}$ Nottingham Trent University
}

\begin{abstract}
The study of memory for texts has had an long tradition of research in psychology. According to most general accounts, the recognition or recall of items in a text is based on querying a memory representation that is built up on the basis of background knowledge. The objective of this paper is to describe and thoroughly test a Bayesian model of these general accounts. In particular, we present a model that describes how we use our background knowledge to form memories in terms of Bayesian inference of statistical patterns in the text, followed by posterior predictive inference of the words that are typical of those inferred patterns. This provides us with precise predictions about which words will be remembered, whether veridically or erroneously, from any given text. We tested these predictions using behavioural data from a memory experiment using a large sample of randomly chosen texts from a representative corpus of British English. The results show that the probability of remembering any given word in the text, whether falsely or veridically, is well predicted by the Bayesian model. Moreover, compared to nontrivial alternative models of text memory, by every measure used in the analyses, the predictions of the Bayesian model were superior, often overwhelmingly so. We conclude that these results provide strong evidence in favour of the Bayesian account of text memory that we have presented in this paper.
\end{abstract}

Keywords: Bayesian models; Computational models; Human Memory; Reconstructive memory; Text memory

Word count: $\approx 15000$

All the code, stimuli, and experimental data described in this paper are freely and openly available. See Appendix A for details.

Correspondence concerning this article should be addressed to Mark Andrews, Department of Psychology, 50 Shakespeare St, Nottingham NG1 4FQ. E-mail: mark.andrews@ntu.ac.uk 


\section{Introduction}

The study of human memory for linguistic materials that go beyond isolated words or sentences - throughout this manuscript, we'll refer to linguistic materials of this nature by the generic term text - has had a long and illustrious history in psychology. The seminal study on this topic is usually attributed to the classic work of Bartlett (1932), while the more contemporary approach began with the now well known works of Bransford and Johnson (1972), Thorndyke (1977), Mandler and Johnson (1977), Kintsch and Van Dijk (1978), Bower, Black, and Turner (1979), and others. The importance of the study of text memory stems, on the one hand, from the fact that coherent pieces of spoken and written language are paradigm examples of naturalistic and ecologically valid stimuli, and as such are well suited to studying how human memory works generally. On the other hand, given that the ultimate cognitive result of language comprehension is a memory representation of the meaning of the text, language comprehension and text memory are inextricably related: understanding text memory facilitates the understanding of language comprehension, and vice versa (see, for example, Zwaan \& Radvansky, 1998; Kintsch, 1998; Van Oostendorp \& Goldman, 1998).

Common to virtually all studies on this topic is the characterization of human memory for text in terms of the integration of the information inherent in the text itself with our background knowledge and experiences, usually described in the form of knowledge schemas. Bartlett (1932) is widely credited with first describing memory in general, and memory for stories in particular, in these terms. For example, as is now well known, in his classic War of the Ghosts study, Bartlett described how memories of a native American folktale were distorted to fit patterns based on the participants' knowledge and experience. Later, studies that followed broadly in Bartlett's tradition, such as Bransford and Franks (1971), Bransford and Johnson (1972), Bransford et al. (1972), Sulin (1974), Barclay (1973), demonstrated that memory for text is more than the memory for the verbatim information and is in fact built up by integrating the information in the text itself with the participants' background knowledge and experiences. With the advent of methods for knowledge representation in artificial intelligence research, such as the work on knowledge frames (e.g., Minsky, 1975) or scripts (e.g., Schank \& Abelson, 1977), similar concepts were used or developed within cognitive psychology to describe the role of background knowledge in memory for text. For example, Bower et al. (1979) described background knowledge of everyday events in terms of prototypical scripts that describe the main objects and series of actions that characterize the events. They showed, like Bartlett, that memory for texts describing these types of events were distorted to match the patterns in the scripts. Likewise, both Mandler and Johnson (1977) and Thorndyke (1977), following the work of Rumelhart (1975), described knowledge of narrative structures in terms of story grammars, with stories being parsed using these grammars such that the resulting memory representations are essentially fits of the story to the structures defined by the grammar. Van Dijk and Kintsch (1983), building on the work of, for example, Kintsch (1974) and Kintsch and Van Dijk (1978), argued that the ultimate outcome of text comprehension is a memory representation of the situation or state of affairs that the text describes. Crucially, this memory representation is built up using background knowledge in the form of schemas. Following from the above mentioned studies, or developed in parallel to it, something close to a broad consensus about the general characteristics of 
a)

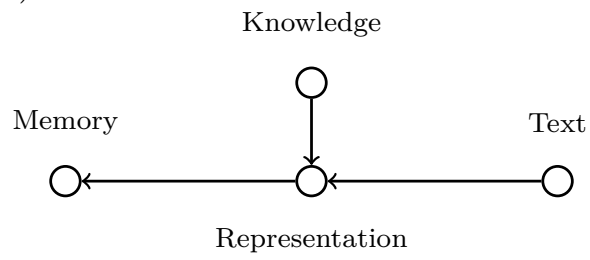

b)

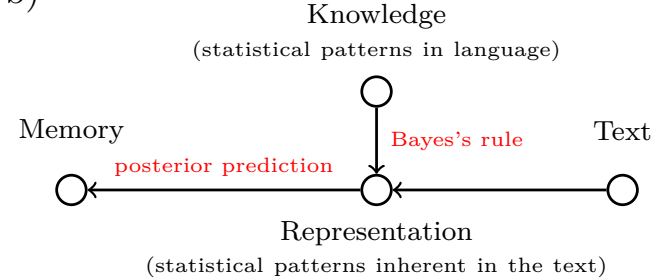

Figure 1. a) A schematic representation of the widely held account of text memory. According to this account, we use our background knowledge to construct a representation of the text's content, and our recall or recognition of words in the text is based on querying this representation. b) A Bayesian computational model version of the general account of text memory. Background knowledge is knowledge of statistical patterns that represent the discourse topics that characterize natural language. The memory representation of the text's content is obtained by inferring, using Bayes's rule, which discourse topics are inherent in that text. Finally, the recall or recognition of words from the text is based on posterior predictive probability that describes which words are and are not typical of topics that were inferred in the text.

text memory has emerged. According to this account, which we summarize by the schematic shown in Figure 1a, the recognition or recall of items in a text is based on querying a memory representation that has been built up by integrating the information in the text itself with background knowledge and experience. This consensual view is evident, either explicitly or implicitly, in the following sample of studies over the past few decades, amongst many others: Garnham (1981), Abbott, Black, and Smith (1985), Kintsch (1988), McKoon and Ratcliff (1992), Graesser, Singer, and Trabasso (1994), McNamara and Kintsch (1996), Zwaan and Radvansky (1998), Brainerd and Mojardin (1998), Kintsch (1998), Schwartz and Bransford (1998), Cain, Oakhill, Barnes, and Bryant (2001), Cook, Limber, and O'Brien (2001), Frank, Koppen, Noordman, and Vonk (2003), Mason and Just (2004), Shapiro (2004), Xu, Kemeny, Park, Frattali, and Braun (2005), Brainerd, Reyna, and Estrada (2006), Frank, Koppen, Noordman, and Vonk (2008), Crocker, Knoeferle, and Mayberry (2010), Van den Broek (2010), Reyna, Corbin, Weldon, and Brainerd (2016), Danvers and Shiota (2017), Richmond and Zacks (2017), Johansson, Oren, and Holmqvist (2018), Yang, Zhang, Yang, and Lin (2018).

Despite being widely endorsed, this general account of text memory is nonetheless a predominantly verbal or informal theory. Its vital characteristics and their putative inter-relations, such as those outlined in Figure 1a, are usually not formally defined. In particular, there is usually no formal or computational account of the nature of background knowledge and how it is represented and acquired, how this knowledge is integrated with the information in the text itself to form a memory representation of the text, nor how this memory representation is queried when recalling or recognizing items from the text. For example, in many cases, background knowledge is described in terms of schemata but without any further specific details or definitions. However, it was apparent to even some of its original advocates that schema theory, as an account of text memory, provides few 
detailed process assumptions ... allows sufficient flexibility to accommodate post hoc many empirical results ... (and) is of limited predictive value and is not testable as a scientific theory (Thorndyke \& Yekovich, 1980, p. 23). Likewise, Alba and Hasher (1983) criticized schema theory, particularly as applied to text memory, as being ill defined and lacking any systematic treatment, and this criticism of the vague nature of schemas in psychology and neuroscience is still valid (see, for example, Ghosh \& Gilboa, 2014). In other cases, such as Schank and Abelson (1977), Rumelhart (1975), Mandler and Johnson (1977) and Thorndyke (1977), where background knowledge was defined in more formal terms, the grammars or other knowledge structure had to be manually coded and were done so for only a limited set of illustrative examples, and the parsing of text content in terms of these grammars or knowledge structures also had to be performed manually. Even ostensibly formal or computational models of knowledge influences on text representation, such as Kintsch (1988), Kintsch (1998), Trabasso and Langston (1998), Van den Broek, Young, Tzeng, Linderholm, and others (1998), Schmalhofer, McDaniel, and Keefe (2002), Trabasso and Bartolone (2003), Tzeng, Van Den Broek, Kendeou, and Lee (2005), also rely heavily or exclusively on either manual parsing of text structures, manual coding of background knowledge schemas and the inference that they lead to, or can can only be applied to small and contrived text examples as opposed to naturalistic text. Consequently, at present, there is no formal or computational account of how background knowledge is used to infer a memory representation of text content that is sufficiently precise to lead to testable empirical predictions using everyday texts. As such, this general account is challenging to test or falsify, or to refine and elaborate as a theory of memory or of cognition generally.

The aim of the present paper is therefore to present a formal theoretical account of text memory in terms of Bayesian inference and prediction, and to test its behavioural predictions with memory experiments using everyday texts. Specifically, we will present a Bayesian computational model that describes the memory representation of a text in terms of statistically inferred patterns in the text, and describe the recognition and recall of words in the text in terms of predictive inferences based on these statistical patterns. This model allows us to make precise empirical predictions concerning the probability that any given word will be recalled or recognized, whether falsely or veridically, from any given text, and we will test these predictions in behavioural studies using a representative sample of everyday English texts.

\section{A Bayesian Model of Text Representation and Memory}

In order to motivate the Bayesian model that we will develop, we begin with the premise, also shared by virtually all of the studies mentioned above, that the memory representation of a text is a representation of its semantic content. We assume that, to a first approximation, the text's semantic content can be described by the set of discourse topics that are instantiated in the text, with each discourse topic being represented by a cluster of inter-related words. As an example, consider the following text:

Despite the challenges associated with building up large-scale production, the Japanese are forging ahead in display technology — and for very good reasons. Obviously the straightforward replacement of bulky cathode ray tubes with flat 
panel display leads to significant power and weight reductions as exemplified in the commercialisation of laptop computers...

It is clear that this text is about, roughly speaking, topics such as industry, technology, and so on. Likewise, a topic such as industry could in general be exemplified by words like plant, company, factory, production, and many others, while a topic such as technology could in general be exemplified by words like computer, electronics, engineering, and many others. In other words, we can approximately describe the semantic content of any given text by identifying within it a set of discourse topics, taken from a potentially large set of discourse topics, with each of these discourse topics being characterized by a possibly large set of semantically inter-related words. Whenever we read or hear a text, we infer its discourse topics by recognizing different clusters of words, each of which signify different topics. These inferred topics then describe our representation of what the text is about. On the basis of this representation, recall or recognition of items from the text is then essentially assessing whether any given item is compatible with, or typical of, the topics that have been inferred in the text. For example, on the basis of the text above, there might be high probability of recognizing or recalling words such as display (which did occur) or monitor (which did not occur) because both are typical of some of the main inferred discourse topics.

To put the foregoing description in somewhat more formal terms, we will denote the set of all possible discourse topics across spoken and written language by $\phi_{1}, \phi_{2} \ldots \phi_{k} \ldots$, with each $\phi_{k}$ being, in general, a probability distribution over all words in the language. Given that these topics are essentially statistical patterns that characterize spoken and written language, they can therefore be inferred (or learned, acquired, etc.) from a language corpus via Bayes's rule. In other words, if $\mathcal{D}$ is a language corpus, using Bayes's rule, as we will explain, we may infer

$$
\mathrm{P}\left(\phi_{1}, \phi_{2} \ldots \phi_{k} \ldots \mid \mathcal{D}\right)
$$

which gives the probability distribution over the topics that characterize $\mathcal{D}$. Having learned the topics that characterise the language, for any particular text, denoted by $t_{j}$, we can then infer, again via Bayes's rule,

$$
\mathrm{P}\left(\pi_{j} \mid t_{j}\right)
$$

where $\pi_{j}$ is a probability distribution over the discourse topics in the language. This is equivalent to performing a statistical parse of the topics in the text, and leads to a representation of what the text is about in terms of a probability distribution over all possible topics. Effectively, therefore, $\pi_{j}$ is the representation of $t_{j}$ in terms of the topics that characterize or typify it. Finally, on the basis of this memory representation, using Bayesian posterior predictive inference, we may predict which words are and are not typical or compatible with the text's inferred topics, and from this we can calculate the probability that any given word will, whether falsely or veridically, be recognized or recalled from the text. Specifically, having inferred $\pi_{j}$, we may then calculate

$$
\mathrm{P}\left(w \mid t_{j}\right)=\int \mathrm{P}\left(w \mid \pi_{j}\right) \mathrm{P}\left(\pi_{j} \mid t_{j}\right) d \pi_{j}
$$

which gives the probability distribution over the words that are predicted or implied by the $\pi_{j}$ representation of text $t_{j}$. 
This description provides the outline of a computational version of the general account of text memory mentioned above. We provide a schematic of this in Figure 1b. It also outlines formal or computational descriptions of the various details that remained vaguely described in the original account. For example, according to this computational account, the necessary background knowledge for text memory is knowledge of statistical patterns in spoken and written language. We acquire knowledge of these patterns via Bayesian learning from the statistics of natural language. Our cognitive or memory representation of any given text is a representation of which topics are inherent in that text, and this is inferred using posterior inference using Bayes's rule. Finally, recognizing or recalling items from a text is based on posterior predictive inference of which words are or are not typical of the topics inferred in the text.

\section{A Hierarchical Dirichlet Process Topic Model}

The Bayesian model described thus far assumes a probabilistic language model. In other words, it assumes that we have explicitly specified a probabilistic model that generates a language corpus, and one that does so specifically in terms of a set of component distributions $\Phi=\phi_{1}, \phi_{2} \ldots \phi_{k} \ldots$, each of which is simply a probability distribution over a vocabulary of words. This language model, by definition, effectively provides a complete account of the probabilistic or statistical structure of language. There are infinitely many probabilistic language models that we could employ here. Some, such as those involving probabilistic grammars, focus on providing accurate descriptions of more fine-grained syntactic structures. Other probabilistic language models, such as probabilistic topic models (see, for example, Griffiths, Steyvers, \& Tenenbaum, 2007; Blei, 2012; Blei, Ng, \& Jordan, 2003; Steyvers \& Griffiths, 2007) have proved highly effective in capturing the statistical patterns that characterize the coarse-grained statistical structures across spoken and written language. Here, we use a type of probabilistic topic model known as a hierarchical Dirichlet process topic model (HDPTM) (Teh, Jordan, Beal, \& Blei, 2004, 2006).

A HDPTM is a bag of words probabilistic language model. According to a bag of words model, a language corpus $\mathcal{D}$ is a set of texts, where each text is an unordered set of words. In other words, within each text, all sequential or syntactic information is ignored and the only information that is used is which words occur and with what frequency. More formally, according to a bag of words model, the corpus of language data $\mathcal{D}$ is treated as a set of $J$ texts $t_{1}, t_{2} \ldots t_{j} \ldots t_{J}$, where each $t_{j}$ is a set of $n_{j}$ words from a finite vocabulary, represented simply by the $V$ integers $\{1,2 \ldots V\}$. In other words, each $t_{j}=w_{j 1}, w_{j 2} \ldots w_{j i} \ldots w_{j n_{j}}$, with each $w_{j i} \in\{1 \ldots V\}$, and so $\mathcal{D}=\left\{w_{j 1}, w_{j 2} \ldots w_{j i} \ldots w_{j n_{j}}\right\}_{j=1}^{J}$.

As a generative model of this corpus, the HDPTM treats each observed word $w_{j i}$ as a sample from one of infinitely many discourse topics $\phi_{1}, \phi_{2} \ldots \phi_{k} \ldots$, where each $\phi_{k}$ is a probability distribution over $\{1 \ldots V\}$. Although each $\phi_{k}$ is a distribution over the entire vocabulary, because it will place most of its probability mass on a relatively small number of words, in effect it identifies a cluster of inter-related words that describe some meaningful discourse topic (see examples of inferred topics on Page 11). The identity of the particular

topic distribution from which each $w_{j i}$ is drawn is determined by the value of a discrete 
latent variable $x_{j i} \in\{1,2 \ldots k \ldots\}$ that corresponds to $w_{j i}$. As such, we model each $w_{j i}$ as

$$
w_{j i} \mid x_{j i}, \phi \sim \operatorname{Categorical}\left(\phi_{\left[x_{j i}\right]}\right),
$$

where Categorical $(\cdot)$ denotes a categorical distribution. The probability distribution over these possible values of $x_{j i}$ is given by by $\pi_{j}$, which is a categorical distribution over the positive integers that is specific to text $t_{j}$. In other words,

$$
x_{j i} \mid \pi_{j} \sim \text { Categorical }\left(\pi_{j}\right) .
$$

Each $\pi_{j}$, in turn, is assumed to be drawn from a Dirichlet process prior (see Ferguson, 1973) whose base distribution, $m$, is a categorical distribution over the positive integers and whose scalar concentration parameter is $a$ :

$$
\pi_{j} \mid a, m \sim \operatorname{DirichletProcess}(a, m),
$$

which is equivalent to

$$
\pi_{j 1}, \pi_{j 2} \ldots \pi_{K}, \sum_{k>K} \pi_{k} \mid a, m \sim \operatorname{Dirichlet}\left(a m_{1}, a m_{2} \ldots a m_{K}, a \sum_{k>K} m_{k}\right),
$$

where Dirichlet( $\cdot)$ is a standard Dirichlet distribution, which is a probability distribution over finite categorical distributions. In other words, the Dirichlet process prior over each $\pi_{j}$ is the infinite dimensional counterpart to a Dirichlet distribution over finite dimensional probability distributions. The $m$ base distribution of the Dirichlet process is assumed to be drawn from a stick-breaking prior (see Ishwaran \& James, 2001) with a parameter $\gamma$ :

$$
m \mid \gamma \sim \operatorname{StickBreaking}(\gamma)
$$

A stick-breaking distribution is a probability distribution over infinite-dimensional categorical distributions, and is the infinite dimensional counterpart of the Generalized Dirichlet Distribution (Connor \& Mosimann, 1969). The prior distributions of the Dirichlet process concentration parameter $a$ and the stick-breaking parameter $\gamma$ are Gamma distributions, both with shape and scale parameters equal to 1 . For the topic distributions, $\phi_{1}, \phi_{2} \ldots \phi_{k} \ldots$, we can assume they are independently and identically drawn from a Dirichlet distribution with a length $V$ location parameter $\psi$ and concentration parameter $b$. In turn, $\psi$ is drawn from a symmetric Dirichlet distribution with concentration parameter $c$ (i.e. $\psi \mid c \sim \operatorname{Dirichlet}\left(c \cdot \mathbf{1}_{V}\right)$, where $c \cdot \mathbf{1}_{V}$ is a length $V$ vector with each element being $c$ ). Finally, both $b$ and $c$, like $a$ and $\gamma$, can be given Gamma priors, again with shape and scale distributions equal to 1 . The full specification of the HDPTM is provided in Figure 2.

Inference. Given any corpus of natural language $\mathcal{D}$, the posterior distribution over all the unobserved variables, which includes all latent variables, parameters, and hyperparameters, in the HDPTM is

$$
\begin{aligned}
& \mathrm{P}\left(\vec{x}_{1: J}, \phi, \pi_{1: J}, \psi, m, a, b, c, \gamma \mid \mathcal{D}\right) \\
& \quad \propto \mathrm{P}(\psi \mid c) \mathrm{P}\left(\pi_{1: J} \mid a, m\right) \mathrm{P}(m \mid \gamma) \mathrm{P}(b, c, a, m) \int \mathrm{P}\left(\mathcal{D} \mid \vec{x}_{1: J}, \phi\right) \mathrm{P}\left(\vec{x}_{1: J} \mid \pi_{1: J}\right) \mathrm{P}(\phi \mid b, \psi) d \vec{x}_{1: J} d \phi
\end{aligned}
$$

where $\vec{x}_{1: J} \doteq\left\{x_{j 1}, x_{j 2} \ldots x_{j n_{j}}\right\}_{j=1}^{J}, \phi \doteq \phi_{1}, \phi_{2} \ldots, \pi_{1: J} \doteq \pi_{1}, \pi_{2} \ldots \pi_{j} \ldots \pi_{J}$. We may draw samples from this high dimensional posterior distribution using a Gibbs sampler. We derived this sampler based on the work of Teh et al. (2004), Teh et al. (2006), Newman, Asuncion, Smyth, and Welling (2009) and we describe it in full technical detail in Andrews (2019a). 

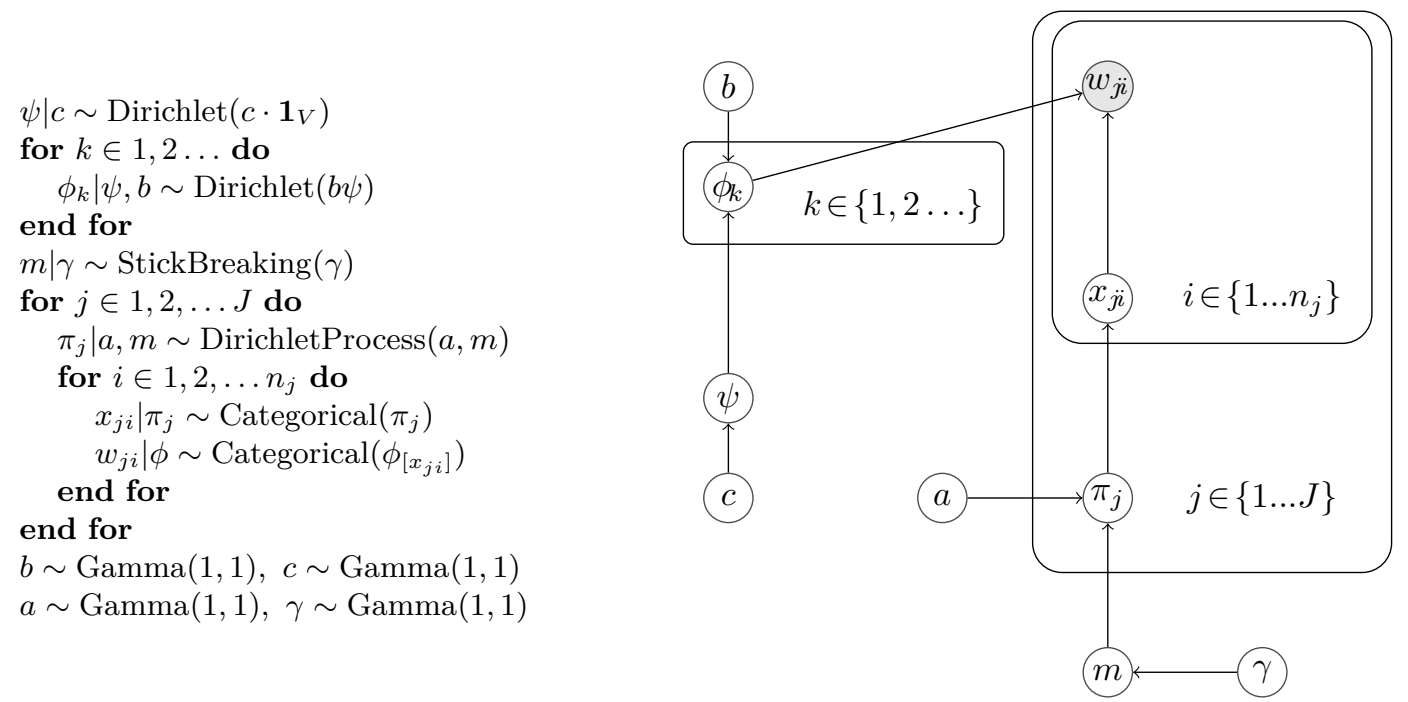

Figure 2. The hierarchical Dirichlet process topic model (HDPTM) is a generative model of a bag of words language corpus. Any bag of words corpus can be specified as a set of $J$ unordered sets of words $\mathcal{D}=\left\{w_{j 1}, w_{j 2} \ldots w_{j n_{j}}\right\}_{j=1}^{J}$. The system of probabilty distributions shown on the left specifies the probabilistic generative model of $\mathcal{D}$ according to the HDPTM. On the right, we provide a so-called Bayesian network that shows all the variables in the HDPTM and their inter-dependencies. The Bayesian network is a directed acyclic graph (DAG) with each node or vertex in the graph being a random variable in the HDPTM. A shaded node indicates that the variable is observed. A directed edge between any two variables indicates that the random variable to which the edge is directed is dependent on the value of the random variable from it orginates. The set of random variables and the directed edges between them in the Bayesian network is completely determined by the system of probability distributions shown on the left.

Posterior predictive distributions. Having inferred the posterior distribution of the unobserved variables in the HDPTM, for any new text $t_{j^{\prime}}$, the posterior predictive distribution over the vocabulary conditioned on $t_{j^{\prime}}$ is, informally speaking, the distribution over words that are consistent with the discourse topics of text $t_{j^{\prime}}$. It is calculated as follows:

$$
\begin{aligned}
\mathrm{P}\left(w \mid \phi, t_{j^{\prime}}, a, m\right) & =\int \mathrm{P}\left(w \mid \phi, \pi_{j^{\prime}}\right) \mathrm{P}\left(\pi_{j^{\prime}} \mid t_{j^{\prime}}, \phi, a, m\right) d \pi_{j^{\prime}} \\
& =\int\left[\sum_{k=1} \mathrm{P}(w \mid \phi, x=k) \mathrm{P}\left(x=k \mid \pi_{j^{\prime}}\right)\right] \mathrm{P}\left(\pi_{j^{\prime}} \mid t_{j^{\prime}}, \phi, a, m\right) d \pi_{j^{\prime}}
\end{aligned}
$$

where $\mathrm{P}\left(\pi_{j^{\prime}} \mid t_{j^{\prime}}, \phi, a, m\right)$ is the posterior distribution over topic distributions for text $t_{j^{\prime}}$. We can sample from the posterior $\mathrm{P}\left(\pi_{j^{\prime}} \mid t_{j^{\prime}}, \phi, a, m\right)$ using a blocked Gibbs sampler that iterates the following two sampling steps:

$$
\begin{aligned}
\vec{x}_{j^{\prime}} & \sim \mathrm{P}\left(\vec{x}_{j^{\prime}} \mid \vec{w}_{j^{\prime}}, \phi, \pi_{j^{\prime}}\right), \\
\pi_{j^{\prime}} & \sim \mathrm{P}\left(\pi_{j^{\prime}} \mid \vec{x}_{j^{\prime}}, a, m\right),
\end{aligned}
$$

where $\vec{w}_{j^{\prime}}=w_{j^{\prime} 1}, w_{j^{\prime}} \ldots w_{j^{\prime} n_{j^{\prime}}}=t_{j^{\prime}}$ and $\vec{x}_{j^{\prime}}=x_{j^{\prime} 1}, x_{j^{\prime} 2} \ldots x_{j^{\prime} n_{j^{\prime}}}$. In the first step, we sample from the posterior distribution over the latent variables that correspond to the words 
in text $t_{j^{\prime}}$. In the second step, we sample from the posterior distribution over $\pi_{j^{\prime}}$ given the values of $\vec{x}_{j^{\prime}}$ sampled in the previous step. The posterior distributions in both of these two steps can be calculated exactly.

\section{Implementation and Simulations}

In order to explore and evaluate the model just described, we implemented it as a Python/Fortran program (Andrews, 2019b) and trained it on a large representative corpus of natural language. The corpus was obtained by extracting paragraphs, or concatenations of consecutive paragraphs, of between 250 and 500 words from the British National Corpus (BNC) (British National Corpus Consortium, 2007). After removing any texts that contained as sub-texts those texts that were used as the stimuli in the behavioural experiment (see next section), this resulted in a set of 183,975 texts of between 250 and 500 words each, with a collective total of over 78 million word tokens. After removing stop words, words that occurred less than 5 times in total in the, and words that did not occur in a standard dictionary of English words ${ }^{1}$, there was a total vocabulary of 49,328 word types in the corpus. See Appendix A for details on the Python code used to create the corpus, and for how to obtain a copy of the corpus itself.

We trained the HDPTM using the corpus just described using the Gibbs sampler. This drew samples from posterior distribution over $\vec{x}_{1: J}, \phi, \pi_{1: J}, \psi, m, a, b, c, \gamma$, for 20,000 iterations. After approximately a few hundred iterations, $99 \%$ of the posterior probability mass converged on approximately 1,340 topics. In other words, after a few hundred iterations

$$
\sum_{k=1}^{K=1340} m_{s_{i}} \approx 0.99,
$$

where $s_{1}, s_{2} \ldots$ are the indices of the elements of $m$ sorted in decreasing order. Samples from iteration 19,000 to 20,000 , thinning by a factor of 10 , were then retained as the final set of posterior samples. Using these posterior samples, for each of held-out text $t_{j^{\prime}}$, we calculated the posterior predictive distributions as follows:

$$
\mathrm{P}\left(w \mid \bar{\phi}, t_{j^{\prime}}, \bar{a}, \bar{m}\right)
$$

where $\bar{\phi}, \bar{a}, \bar{m}$ are the means of the posterior samples of $\phi, a, m$, respectively.

\section{How the Bayesian Model of Text Memory Works}

To reiterate, the contention of this paper is that the widely held, yet arguably vague and untestable, account of memory for text (see Figure 1a) can be described formally as a Bayesian computational model (see Figure 1b), and in practice we can instantiate this model using a HDPTM. Having described the HDPTM, we may now describe how it can act as a computational model of human memory for text. There are three main components to the model. First, there is the representation of the background knowledge that is relevant for constructing memory representations. Second, there is how this background knowledge is

\footnotetext{
${ }^{1}$ We used the 2of 4 brif.txt word list from the 12Dicts collection of English word lists, available at http://wordlist.aspell.net/12dicts.
} 
used, via Bayes's rule, to infer a representation of a text's semantic content. This is effectively the text's memory representation. Finally, having inferred the text's representation, the recognition or recall of the word's in the text is based on the posterior predictive distribution over words conditional of the text's representation.

The model assumes that background knowledge, or at least the background knowledge that is most relevant for inferring a text's semantic content, is represented by a large set of discourse topics $\phi_{1}, \phi_{2} \ldots$. These are assumed to be learned over the course of our lives from experience with the statistics of spoken and written language, and are the Bayesian model's counterparts of the schemata or scripts, etc., in the widely held account of text memory. Examples of topics inferred by the HDPTM trained on the corpus described above are illustrated in Table 1. In each case, we display the 10 most probable words ${ }^{2}$ in each topic. In general in a HDPTM or any other probabilistic topic models, each topic often identifies some intuitively meaningful discourse topic that could perhaps be provided with a meaningful approximate label such as, for some of the examples shown, university, home furniture, Northern Ireland, and so on. Although there are in principle infinitely many topics in a HDPTM, in any given model, the posterior distribution will be concentrated on a relatively small number of topics. For any medium sized corpus of natural language, we would expect the posterior distribution to be concentrated on at least many hundreds, though possibly thousands, of topics like those shown in the table. In the simulations that we use in this paper, as mentioned above, approximately $99 \%$ of the posterior distribution over the topics is concentrated on around 1340 distinct topics.

Having learned the repertoire of discourse topics, we can infer the probability distribution over the topics in any new text $t_{j^{\prime}}$ using Bayes's rule: $\mathrm{P}\left(\pi_{j^{\prime}} \mid t_{j^{\prime}}, \phi, a, m\right)$. Here, $\pi_{j^{\prime}}$ is a probability distribution over all topics and can be seen as the semantic representation of text $t_{j^{\prime}}$ : it tells us which topics are instantiated in text $t_{j^{\prime}}$ and with what probabilities. In other words, it provides a semantic parse of text $t_{j^{\prime}}$ in terms of the repertoire of discourse topics. As an example, consider the following text.

Individual goals may well be in conflict with organisational goals even where there is a strong commitment to one goal by the organisation. It is worth repeating that there may also be competing goals within the organisation itself. Thus, for example, the prison service may wish to pursue the twin goals of the punishment of offenders and at the same time their rehabilitation. These two goals may prove to be incompatible and yet each will have its supporters. Within the National Health Service there may well be a commitment to provide the best possible medical care that the state of medical knowledge will allow and yet this may be tempered by the requirement to act within tight financial constraints. The two goals may be in conflict. Many organisations will face the problem of which goals to prioritise in the light of competing goals.

Using the averages of the samples of the posterior of $\phi, a, m$, which we denote by $\bar{\phi}, \bar{a}$, and $\bar{m}$, respectively, we can then draw samples from $\mathrm{P}\left(\pi_{j^{\prime}} \mid t_{j^{\prime}}, \bar{\phi}, \bar{a}, \bar{m}\right)$, the average of which

\footnotetext{
${ }^{2}$ While each topic is always defined by a probability distribution over the entire vocabulary of $V$ words, in most topics the probability mass is concentrated on a relatively small subset of words, and this allows us to illustrate topics by displaying only their most probable words.
} 


\begin{tabular}{|c|c|c|c|c|c|c|}
\hline $\begin{array}{l}\text { evil } \\
\text { life } \\
\text { death } \\
\text { sin } \\
\text { god } \\
\text { truth } \\
\text { human } \\
\text { true } \\
\text { guilt } \\
\text { moral }\end{array}$ & $\begin{array}{c}\text { cells } \\
\text { cell } \\
\text { membrane } \\
\text { receptor } \\
\text { calcium } \\
\text { activity } \\
\text { shown } \\
\text { protein } \\
\text { response } \\
\text { increased }\end{array}$ & $\begin{array}{l}\text { patients } \\
\text { gastric } \\
\text { ulcer } \\
\text { duodenal } \\
\text { acid } \\
\text { gastritis } \\
\text { secretion } \\
\text { subjects } \\
\text { healing } \\
\text { ulcers }\end{array}$ & $\begin{array}{c}\text { environmental } \\
\text { pollution } \\
\text { environment } \\
\text { emissions } \\
\text { air } \\
\text { sulphur } \\
\text { dioxide } \\
\text { carbon } \\
\text { acid } \\
\text { rain }\end{array}$ & $\begin{array}{c}\text { aids } \\
\text { virus } \\
\text { infection } \\
\text { disease } \\
\text { infected } \\
\text { diseases } \\
\text { hepatitis } \\
\text { blood } \\
\text { people } \\
\text { health }\end{array}$ & $\begin{array}{l}\text { difficult } \\
\text { solution } \\
\text { difficulties } \\
\text { situation } \\
\text { future } \\
\text { difficulty } \\
\text { remain } \\
\text { task } \\
\text { question } \\
\text { alternative }\end{array}$ & $\begin{array}{c}\text { questions } \\
\text { question } \\
\text { answer } \\
\text { answers } \\
\text { answered } \\
\text { answering } \\
\text { information } \\
\text { reply } \\
\text { difficult } \\
\text { simple }\end{array}$ \\
\hline $\begin{array}{l}\text { music } \\
\text { orchestra } \\
\text { recording } \\
\text { piano } \\
\text { symphony } \\
\text { performance } \\
\text { recordings } \\
\text { playing } \\
\text { composer } \\
\text { sound }\end{array}$ & $\begin{array}{l}\text { football } \\
\text { club } \\
\text { league } \\
\text { players } \\
\text { game } \\
\text { team } \\
\text { cup } \\
\text { season } \\
\text { manager } \\
\text { fans }\end{array}$ & $\begin{array}{c}\text { book } \\
\text { published } \\
\text { books } \\
\text { guide } \\
\text { author } \\
\text { history } \\
\text { written } \\
\text { volume } \\
\text { edition } \\
\text { information }\end{array}$ & $\begin{array}{l}\text { words } \\
\text { word } \\
\text { meaning } \\
\text { language } \\
\text { meanings } \\
\text { phrase } \\
\text { means } \\
\text { sentence } \\
\text { dictionary } \\
\text { phrases }\end{array}$ & $\begin{array}{l}\text { guitar } \\
\text { sound } \\
\text { bass } \\
\text { guitars } \\
\text { neck } \\
\text { amp } \\
\text { sounds } \\
\text { playing } \\
\text { strings } \\
\text { tone }\end{array}$ & $\begin{array}{c}\text { forest } \\
\text { soil } \\
\text { land } \\
\text { conservation } \\
\text { erosion } \\
\text { forests } \\
\text { tropical } \\
\text { environmental } \\
\text { timber } \\
\text { rainforest }\end{array}$ & $\begin{array}{c}\text { army } \\
\text { battle } \\
\text { soldiers } \\
\text { enemy } \\
\text { miles } \\
\text { king } \\
\text { horses } \\
\text { horse } \\
\text { troops } \\
\text { time }\end{array}$ \\
\hline $\begin{array}{l}\text { education } \\
\text { schools } \\
\text { school } \\
\text { children } \\
\text { educational } \\
\text { teachers } \\
\text { secondary } \\
\text { pupils } \\
\text { system } \\
\text { parents }\end{array}$ & $\begin{array}{c}\text { fish } \\
\text { tank } \\
\text { water } \\
\text { filter } \\
\text { aquarium } \\
\text { species } \\
\text { plants } \\
\text { tanks } \\
\text { fry } \\
\text { pond }\end{array}$ & $\begin{array}{c}\text { roof } \\
\text { tiles } \\
\text { wall } \\
\text { walls } \\
\text { timber } \\
\text { floor } \\
\text { wood } \\
\text { damp } \\
\text { insulation } \\
\text { building }\end{array}$ & $\begin{array}{l}\text { species } \\
\text { fossil } \\
\text { fossils } \\
\text { dinosaurs } \\
\text { animals } \\
\text { living } \\
\text { mammals } \\
\text { animal } \\
\text { preserved } \\
\text { rocks }\end{array}$ & $\begin{array}{c}\text { business } \\
\text { market } \\
\text { company } \\
\text { sales } \\
\text { growth } \\
\text { future } \\
\text { increased } \\
\text { markets } \\
\text { management } \\
\text { major }\end{array}$ & $\begin{array}{c}\text { music } \\
\text { musical } \\
\text { musicians } \\
\text { choir } \\
\text { church } \\
\text { singing } \\
\text { songs } \\
\text { composers } \\
\text { instruments } \\
\text { organ }\end{array}$ & $\begin{array}{l}\text { college } \\
\text { university } \\
\text { oxford } \\
\text { professor } \\
\text { school } \\
\text { students } \\
\text { royal } \\
\text { lecturer } \\
\text { academic } \\
\text { student }\end{array}$ \\
\hline $\begin{array}{c}\text { shares } \\
\text { company } \\
\text { share } \\
\text { shareholders } \\
\text { capital } \\
\text { issue } \\
\text { stock } \\
\text { ordinary } \\
\text { rights } \\
\text { issued }\end{array}$ & $\begin{array}{l}\text { northern } \\
\text { unionist } \\
\text { paisley } \\
\text { political } \\
\text { government } \\
\text { catholic } \\
\text { loyalist } \\
\text { province } \\
\text { unionists } \\
\text { republic }\end{array}$ & $\begin{array}{l}\text { service } \\
\text { services } \\
\text { provide } \\
\text { customers } \\
\text { range } \\
\text { offer } \\
\text { customer } \\
\text { providing } \\
\text { support } \\
\text { provided }\end{array}$ & $\begin{array}{c}\text { module } \\
\text { package } \\
\text { lifespan } \\
\text { modules } \\
\text { user } \\
\text { version } \\
\text { manager } \\
\text { approval } \\
\text { access } \\
\text { users }\end{array}$ & $\begin{array}{c}\text { debt } \\
\text { loan } \\
\text { loans } \\
\text { finance } \\
\text { money } \\
\text { debts } \\
\text { bank } \\
\text { capital } \\
\text { pay } \\
\text { borrowing }\end{array}$ & $\begin{array}{c}\text { nuclear } \\
\text { weapons } \\
\text { arms } \\
\text { soviet } \\
\text { treaty } \\
\text { defence } \\
\text { missiles } \\
\text { military } \\
\text { disarmament } \\
\text { chemical }\end{array}$ & $\begin{array}{c}\text { road } \\
\text { roads } \\
\text { traffic } \\
\text { bridge } \\
\text { route } \\
\text { transport } \\
\text { bypass } \\
\text { motorway } \\
\text { junction } \\
\text { department }\end{array}$ \\
\hline $\begin{array}{c}\text { age } \\
\text { disease } \\
\text { mortality } \\
\text { incidence } \\
\text { study } \\
\text { rates } \\
\text { population } \\
\text { prevalence } \\
\text { table } \\
\text { data }\end{array}$ & $\begin{array}{c}\text { government } \\
\text { national } \\
\text { president } \\
\text { political } \\
\text { opposition } \\
\text { assembly } \\
\text { party } \\
\text { elections } \\
\text { parties } \\
\text { democratic }\end{array}$ & $\begin{array}{l}\text { poem } \\
\text { poetry } \\
\text { poet } \\
\text { poems } \\
\text { verse } \\
\text { writing } \\
\text { lines } \\
\text { written } \\
\text { poets } \\
\text { poetic }\end{array}$ & $\begin{array}{c}\text { photographs } \\
\text { photograph } \\
\text { photography } \\
\text { pictures } \\
\text { photographer } \\
\text { camera } \\
\text { photographic } \\
\text { picture } \\
\text { photographers } \\
\text { photo }\end{array}$ & $\begin{array}{l}\text { college } \\
\text { university } \\
\text { oxford } \\
\text { professor } \\
\text { school } \\
\text { students } \\
\text { royal } \\
\text { lecturer } \\
\text { academic } \\
\text { student }\end{array}$ & $\begin{array}{c}\text { furniture } \\
\text { table } \\
\text { house } \\
\text { walls } \\
\text { floor } \\
\text { wall } \\
\text { chairs } \\
\text { kitchen } \\
\text { white } \\
\text { glass }\end{array}$ & $\begin{array}{l}\text { women } \\
\text { male } \\
\text { female } \\
\text { woman } \\
\text { feminist } \\
\text { gender } \\
\text { feminism } \\
\text { sex } \\
\text { feminists } \\
\text { sexual }\end{array}$ \\
\hline
\end{tabular}

Table 1

Examples of topics inferred by the HDPTM trained on the BNC. In each case, the 10 most probable words in the topic are shown. 
we'll denote by $\bar{\pi}_{j^{\prime}}$. In the following table, we show the 7 most probable topics and their probabilities.

\begin{tabular}{ccccccc}
\hline 0.31 & 0.12 & 0.12 & 0.09 & 0.06 & 0.05 & 0.03 \\
\hline objectives & conflict & organisation & sentence & health & health & punishment \\
goals & conflicts & management & court & care & care & death \\
objective & relationship & organisations & offenders & services & medical & penalty \\
achieve & tensions & structure & courts & patients & services & capital \\
goal & individual & authority & criminal & service & people & crime \\
set & relationships & organisational & prison & practice & education & corporal \\
achieved & situation & managers & justice & practitioners & disease & penal \\
aims & view & responsibility & offences & authorities & illness & criminal \\
means & resolve & control & sentences & practices & national & law \\
aim & issue & individual & offence & hospital & medicine & abolition \\
\hline
\end{tabular}

This shows that this text is represented as being about a mixture of topics, including those that might be labelled objectives (with probability 0.31), conflict (0.12), organization (0.12), and so on.

Finally, having inferred the distribution over the topics of text $t_{j^{\prime}}$, the recognition or recall of words from this text is based on the posterior predictive distribution over words conditional on $\pi_{j^{\prime}}$. If $\tilde{\pi}_{j^{\prime}}^{1}, \tilde{\pi}_{j^{\prime}}^{2}, \ldots \tilde{\pi}_{j^{\prime}}^{t} \ldots \tilde{\pi}_{j^{\prime}}^{t}$ are $T$ samples from the posterior distribution $\mathrm{P}\left(\pi_{j^{\prime}} \mid t_{j^{\prime}}, \bar{\phi}, \bar{a}, \bar{m}\right)$, then the posterior predictive distribution over the vocabularly is given by the following.

$$
\begin{aligned}
\mathrm{P}\left(w \mid \bar{\phi}, t_{j^{\prime}}, \bar{a}, \bar{m}\right) & \approx \frac{1}{T} \sum_{t=1}^{T} \mathrm{P}\left(w \mid \bar{\phi}, \tilde{\pi}_{j^{\prime}}^{t}\right) \\
& =\frac{1}{T} \sum_{t=1}^{T}\left[\sum_{k=1} \mathrm{P}(w \mid \bar{\phi}, x=k) \mathrm{P}\left(x=k \mid \tilde{\pi}_{j^{\prime}}^{t}\right)\right]
\end{aligned}
$$

Illustrations of posterior predictive distributions for some example held out texts, which we then use in the behavioural experiments described next, are shown in Figure 3. In each case, the posterior predictive distribution is a distribution over the entire vocabulary of words, and tells us how much any given word is compatible with, or typical of, the topic-based representation of the text. From this distribution, we have a model of what words will and will not be remembered, whether veridically or falsely, from any given text.

\section{Experimental Evaluation of the Bayesian Model}

In order to properly evaluate this Bayesian account of memory, we will assess how well this model can predict participants' recognition and recall of words from everyday texts. Specifically, we will use randomly selected texts from the BNC that participants read at a normal reading pace, after which their memory is tested using either a recognition or recall test. We then can statistically evaluate how well the Bayesian model predicts what participants do and do not remember, whether veridicaly or falsely, from these texts. 
It was a great discovery. But it was utterly disastrous for classical physics. An electron encircling a nucleus is continuously subject to acceleration as its velocity keeps on changing direction. (Acceleration is just the rate of change of velocity.) It is an inexorable...... atom would be unstable, as its electrons spiralled ever closer to the nucleus, and it would emit its dying radiation in a band of frequencies with no trace of discreteness. The nuclear atom dealt the coup de grace to classical physics.S

Some social scientists have conceptualised these workers in terms of a reserve army of labour. In this case, they would constitute a pool of labour which can be utilised in boom periods and disregarded in recessions. This view sees the experience of the Third world ...... function of the reserve army of labour was keeping wages low and, to this end, the absolute size of the reserve army need not be very great. The high numbers and continuing growth of this sector in Latin America would suggest that concepts of European analysis are not sufficient.

When John Major talks of a 'nation being at ease with itself', which particular nation is he speaking about? Is he addressing his comments to all those who have become marginalised and dispossessed as a result of right-wing Conservative policies over the years? Or is he speaking about that nation of complacent and indifferent shopkeepers ..... capitalist market like a silk glove. England became one nation again, a land of hope and glory, Elgar, teacakes and cricket. This England is not one recognised by many of my friends.

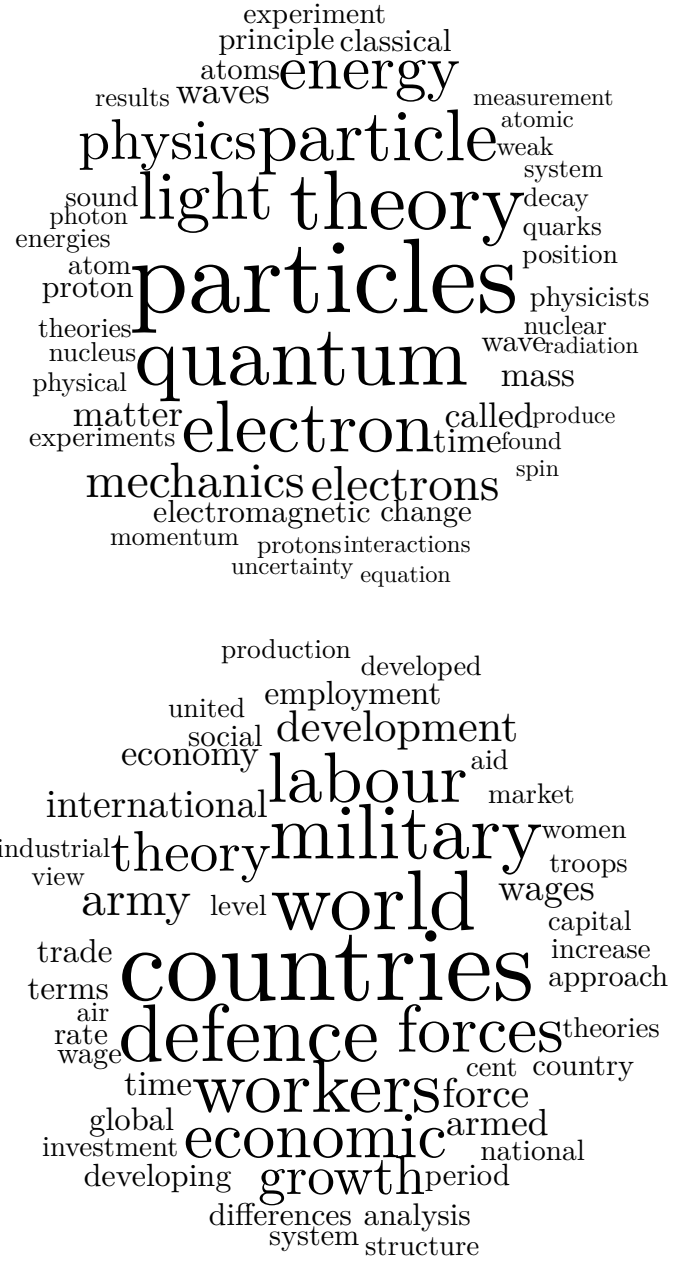

support election conservatives policies language human $c$ conservative political tree political WOr $\mathrm{d}_{\text {public }}$ speech party power

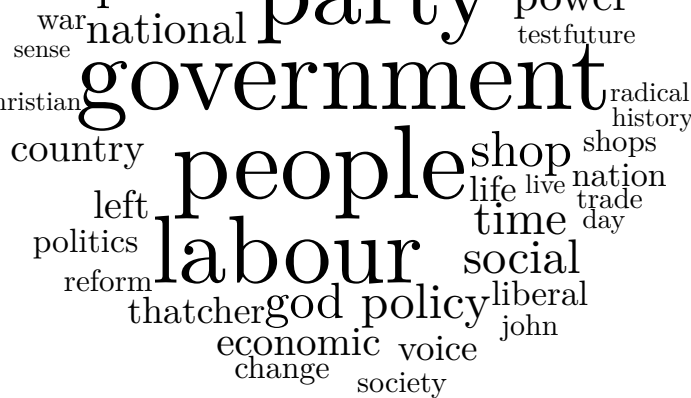

Figure 3. On the left column are shown portions of three example texts used in the behavioural experiments. In the right hand column, corresponding to each text, we show HDPTM's posterior predictive probability distribution over the vocabulary. In each case, the word cloud displays the 50 most probable words according to the posterior distribution, and it scales each word according to its probability. Note that many of the words with relatively high posterior predictive probability may not have actually occurred per se in the corresponding text. To the extent that the posterior predictive distribution is the basis of memory recall and recognition, these items effectively represent false memories. 


\section{Method}

In overview, in this experiment, each participant read a number of separate texts, which were randomly selected from a pool of different texts. After reading each text at normal reading pace, and after a 1 minute filler task, they were then given either a recognition or a recall memory test.

Design and sample size determination. The purpose of the experiment was to obtain sufficient behavioural data to thoroughly test the predictions of the Bayesian model (as well as alternative models for the purposes of comparison; see below). Prior to collecting any data, we conducted numerical simulations to determine the total number of participants, the total number of different memory tests (i.e., different texts followed by recognition or recall tests), and the number of memory tests that any one participant should perform. These simulations demonstrated that to obtain a high probability of detecting the true predictive effect of the model, even if it is assumed to have a low effect size, we required at least 150 participants, at least 50 separate tests, but that each participant need perform only three separate tests. These simulations are described in more detail in Appendix B.

Participants. A total of 216 participants (113 female, 103 male; the median age was 34, with $95 \%$ of participants having ages between 19 and 71) took part in the experiment. Participants were recruited on an entirely voluntary basis from the student population at Nottingham Trent University and elsewhere, with the only restriction being that they were native English speakers.

Materials. A total of 50 texts were used as the to-be-memorized materials in the memory experiment. These were randomly sampled from the BNC subject to the constraint that they were between 140 and 160 words in length, had a high density (over 90\%) of words from a standard dictionary of English words (see Footnote 1), and had a relatively high density (over $75 \%$ ) of words that occurred as stimulus words in the Small World of Words word association norms ${ }^{3}$. We required texts that had a high density of words from a standard dictionary of English words in order to avoid texts on obscure topics with many jargon terms. We required texts with relatively high density of words from the word association norms for purposes of comparing the predictions of the Bayesian model to that of models based on word associations. We will return to this in detail below.

For each of the 50 texts, we produced a recognition memory test word list consisting of 20 words. Of these 20 words, 10 occurred in the text (we will call these the target words) and $10 \mathrm{did}$ not (we'll call these the lure words). The 10 target words were the 10 words in the text that had the highest term-frequency by inverse document frequency (TF-IDF) values. Using TF-IDF values is a simple and widely used method to extract keywords from a text ${ }^{4}$. For the lure words, it is necessary to use words that are not trivially easy to dismiss as not being in the text, as this would largely invalidate the recognition test. To avoid this, the 10 lure words were the 10 words in adjacent paragraphs to the text that had the highest TF-IDF scores, after removing any words that were in the text iself. In other words, given any

\footnotetext{
${ }^{3}$ https://smallworldofwords.org/en/project/home

${ }^{4}$ Informally speaking, words with high TF-IDF values are frequent in the text but do not occur frequently in all texts. More precisely, for any word $w_{i}$, its TF-IDF value is $f_{i} \log \left(N / n_{i}\right)$, where $f_{i}$ is its raw frequency count in the text, $n_{i}$ is number of documents in the corpus where word its occurs at least once, and $N$ is the total number of documents in the corpus.
} 


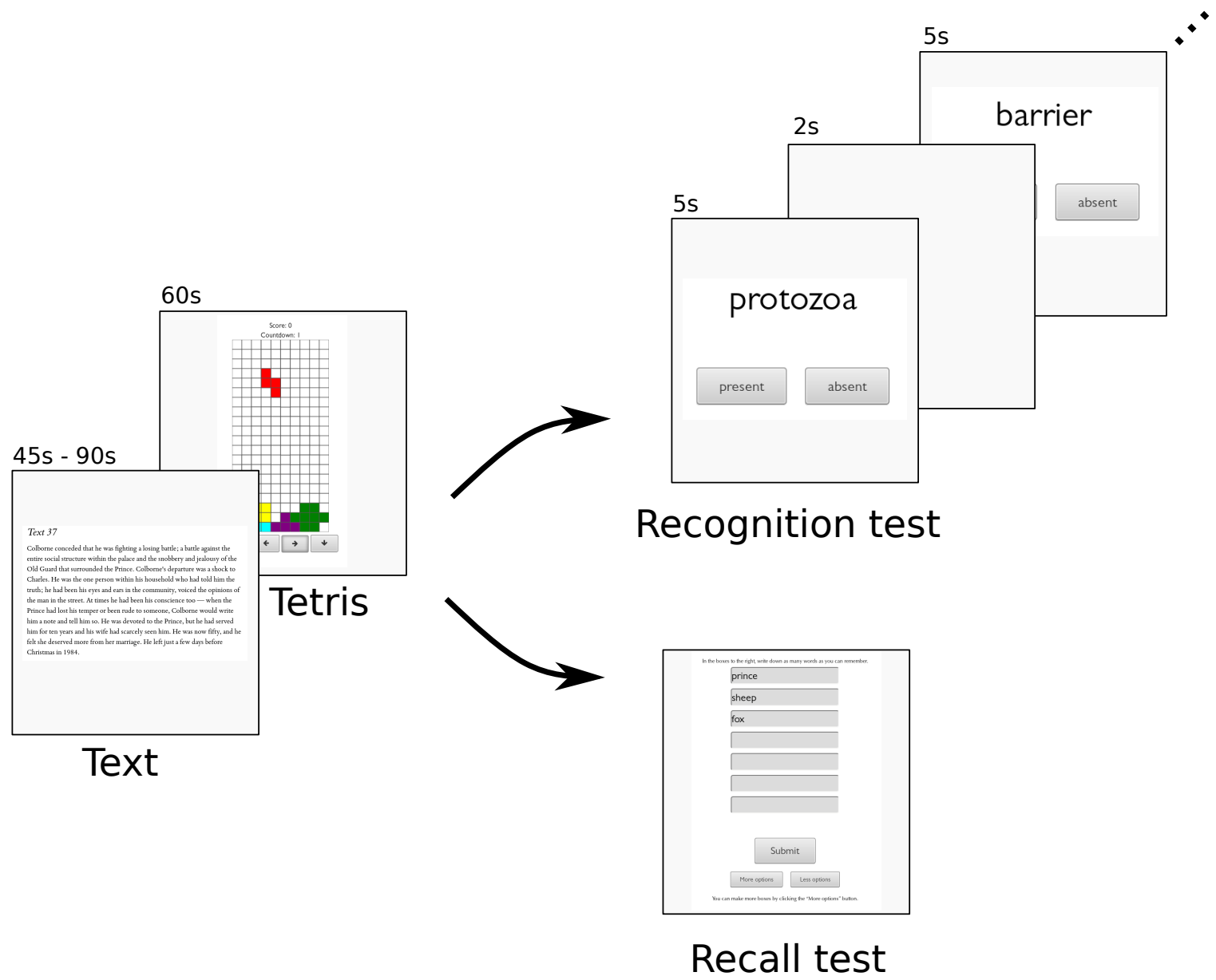

Figure 4. The task diagram of one block in the experiment: Participants read a randomly assigned text, performed a filler task, and then had their memory tested using either a recognition or recall test, with the test type being randomly chosen. This process is repeated three times for each participant.

text, we obtain the paragraph before and after this text as it occurs in the BNC. We then found the words with the 10 highest TF-IDF scores in these two paragraphs combined, after removing any words that occurred in the target text. Given that neighbouring paragraphs will usually be semantically related to the text, these lure words will usually be a set of words that are semantically related to the text, though do not occur in it.

Procedure. For each participant, each experiment session proceeded as follows (see Figure 4 for the task diagram of the experiment). After initial information and instructions, which informed participants that they would be engaging in memory tasks, one of the sample texts appeared on screen. Participants were instructed to read this text at their normal reading pace. The text stayed on screen for a maximum of 90 seconds, but after 45 seconds, participants were able to move on to the next screen if they so wished. On the following screen, participants were asked to play the computer game Tetris for exactly 60 seconds. At the completion of the game, participants proceeded to the memory task. For each participant 
and for each text, the memory test was randomly chosen to be either a recognition or a recall task. For the recognition test, the 20 test items were presented on screen, one word at a time, with an inter-stimulus-interval of 2 seconds. They remained on screen for 5 seconds or until the subject indicated with a button press whether the word shown was present or absent from the text. No feedback was given after each response. If the participant was assigned to the recall test, a screen of a list of small empty text boxes was presented where and they were asked to type as many words as they could remember, one word into each text box. Initially, 10 empty texts boxes were presented, and more boxes could be added with a button press. Upon completion of the memory test, participants were given the option of pausing or proceeding to the next test. Each participant performed up to three tests in total, with the three texts to which they were assigned being always randomly sampled from the set of 50 texts.

As participants were not compensated or otherwise provided incentives to complete all three experimental sessions, not all 216 participants completed three sessions. In total, across all 216 participants, there were 565 separate memory test sessions. Of these 565 separate tests, 289 were recognition memory tests (performed by 182 participants), and 276 were recall memory tests (performed by 174 participants). In general, the average number of tests done per participant was 2.60 sessions.

All experiment sessions were presented using the Wilhelm web-browser based experiment presentation software (Andrews, 2019c) that was hosted at http://www.cognitionexperiments.org. This software allowed the experiment to be done on any web-browser based device, e.g., phones, tablets, laptops and desktops.

\section{Comparison Models}

To meaningfully evaluate the Bayesian model's predictions, it is necessary to compare them to those of nontrivial alternative models. In particular, we will compare the predictions of the Bayesian model to predictions made by two associative models of text memory. Both of these models predict that the words that are remembered, whether falsely or veridically, from any given text are those that are most associated, on average, with the text's content. In other words, they predict that what we do an do not remember from a text is based on the average associative strength between the words in the text and any other word. For example, consider the text from which the following fragement is taken (displayed at greater length in Figure 3 on Page 13):

Some social scientists have conceptualised these workers in terms of a reserve army of labour. In this case, they would constitute a pool of labour which can be utilised in boom periods and disregarded in recessions. This view sees the experience of the Third world ......

According to the associative account of text memory, if words like military, economic, or labour, for example, are highly associated, on average, with words in this text, they have a high probability of being remembered after reading it. Of course, if either of military, economic, or labour, are not, in fact, in the text, then memories of these words would obviously be false memories. This account is precisely the dominant explanation of the Deese-Roediger-McDermott (DRM) effect of false memories from word lists: the word list's 
so-called critical lure word is falsely remembered, according to the dominant account (see, Roediger \& McDermott, 1995; Gallo, 2006, and elsewhere; Roediger, Watson, McDermott, \& Gallo, 2001; Schacter, Guerin, \& Jacques, 2011) because it is highly associated with other words in the list, even though is not present itself. More generally, this dominant account holds that the false memory of the critical lure is merely an accidental byproduct of how memory works normally, which is by forming and remembering associations based on what we read or hear (see Roediger \& McDermott, 2000; Gallo, 2010). Although the DRM effect applies to word lists, its dominant explanation can arguably be extended to memory for texts as well (see Dewhurst, Pursglove, \& Lewis, 2007; Howe \& Wilkinson, 2011).

Association based models are very appropriate choices of models to which we can compare the Bayesian model. On the one hand, they are not trivial or theoretical strawmen models. As just mentioned, they are in fact used as the dominant explanation of highly related memory phenomenon to the one at hand. On the other hand, they are theoretically distinct from the knowledge based accounts of text memory of which the Bayesian model is a computational implementation. The main distinction between knowledge based accounts of memory and associationist ones is that in the former, memories are ultimately based on the meaning of text, while in the latter, they are based on meaning-less associations (Reyna et al., 2016).

Associative accounts of text memory may be operationalized in different ways. Here, we use two operationalisations that differ depending on the precise definition of "association". One approach defines association in terms of statistical cooccurrences of words in spoken and written language. Another approaches defines association in terms of word association norms.

The statistical co-occurrence probability of two words, $w_{k}$ and $w_{l}$, which we will denote $\mathrm{P}_{\mathrm{c}}\left(w_{k}, w_{l}\right)$, is defined as the empirical probability of observing word $w_{k}$ and $w_{l}$ in the same text in the language. Here, we calculate $\mathrm{P}_{\mathrm{C}}\left(w_{k}, w_{l}\right)$ using the same BNC corpus as was used above. From this, we can calculate

$$
\mathrm{P}_{\mathrm{C}}\left(w_{k} \mid w_{l}\right)=\frac{\mathrm{P}_{\mathrm{C}}\left(w_{k}, w_{l}\right)}{\mathrm{P}_{\mathrm{C}}\left(w_{l}\right)}
$$

which is the conditional probability of observing $w_{k}$ in any text given that $w_{l}$ has been observed. From this, if text $t_{j^{\prime}}=w_{j^{\prime} 1}, w_{j^{\prime} 2} \ldots w_{j^{\prime} n_{j^{\prime}}}$, the predicted association probability of word $w_{k}$ according to $t_{j^{\prime}}$ is

$$
\mathrm{P}_{\mathrm{C}}\left(w_{k} \mid t_{j^{\prime}}\right)=\frac{1}{n_{j^{\prime}}} \sum_{i=1}^{n_{j^{\prime}}} \mathrm{P}_{\mathrm{C}}\left(w_{k} \mid w_{j^{\prime} i}\right) .
$$

We can interpret this value intuitively as the average association between $w_{k}$ and $t_{j^{\prime}}$, with association defined in terms of statistical co-occurrences in the language.

An alternative means to calculate the average association between $w_{k}$ and $t_{j^{\prime}}$ is using word association norms, rather than statistical co-occurrences. If $A_{k l}$ is the frequency that word $w_{k}$ is stated as associated with word $w_{l}$, then the conditional probability of word $w_{k}$ 
given $w_{l}$ is

$$
\mathrm{P}_{\mathrm{A}}\left(w_{k} \mid w_{l}\right)=\frac{A_{k l}}{\sum_{i=1}^{V} A_{i l}},
$$

where $V$ is the total number of words in our vocabulary of response words. Now, given $t_{j^{\prime}}=w_{j^{\prime} 1}, w_{j^{\prime} 2} \ldots w_{j^{\prime} n_{j^{\prime}}}$, we can calculate

$$
\mathrm{P}_{\mathrm{A}}\left(w_{k} \mid t_{j^{\prime}}\right)=\frac{1}{n_{j^{\prime}}} \sum_{i=1}^{n_{j^{\prime}}} \mathrm{P}_{\mathrm{A}}\left(w_{k} \mid w_{j^{\prime} i}\right),
$$

which we can interpret as the average association between $w_{k}$ and $t_{j^{\prime}}$, with association now defined in terms of word association norms rather than statistical co-occurrences. Though a large set of English word association norms are available from the widely used Nelson norms (e.g., Nelson, McEvoy, \& Schreiber, 2004), we used an even larger set that is a pre-release of the English small world of words association norms (De Deyne \& Storms, 2017). This provides word associates, produced by 101,119 participants, to 10,050 word types. For more information on how we calculated the predictions according to these associative models, including links to all the source code that was used, see Appendix A.

\section{Results}

Recognition memory data analyses. We begin by analysing the recognition memory test data. In each recognition test, the participant was presented with a list of 20 words, shown one word at a time. Their task was to judge whether each word was either present or absent in the text that they had just read. Across all tests, the average response rate was $97 \%$, and the average accuracy was $76 \%$, with the false positive rate being $28 \%$ (with over $92 \%$ of the 289 tests having a false positive rate greater than 10\%), and the false negative rate being $22 \%$ (with over $92 \%$ of tests having a false negative rate greater than $10 \%)$.

The primary aim of our analysis is to evaluate how well each of our three computational models of memory predict the recognition memory responses. To begin, we label the entire set of responses as $y_{1}, y_{2} \ldots y_{n}$, where $n$ is the total number of recognition test trials across all separate tests and all participants. Each $y_{i}$ takes on the value of either 0 or 1 , where $y_{i}=0$ means that the participant on trial $i$ judged the word shown on that trial, which we label $w_{i}$, to not have been present in the preceding text, while if $y_{i}=1$, the participant judged $w_{i}$ to have been present in the preceding text. For each trial $i$, we also have $s_{i} \in 1 \ldots 216$ and $t_{i} \in 1 \ldots 50$, which identify the participant and the memory test, respectively, corresponding to the $i$ th observation. In addition, we have three predictors of the value of $y_{i}$, namely $\psi_{\left(t_{i}, w_{i}\right)}^{\text {Bayesian }}, \psi_{\left(t_{i}, w_{i}\right)}^{\text {Coocurrence }}$, and $\psi_{\left(t_{i}, w_{i}\right)}^{\text {Associative }}$. These give the predicted probability that $w_{i}$ was in the text of memory test $t_{i}$ according to the Bayesian, the Cooccurrence, and the Associative models, respectively. Naturally, if any one of these predictors is predictive of the behavioural responses, the higher its value, the higher the probability that $y_{i}=1$, and the lower its value, the higher the probability that $y_{i}=0$.

We begin by exploring how well the probabilities according to each memory model correspond to the probabilities of recognizing the words in each memory test. First, separately 
a

test word in text: -- no $\multimap$ yes
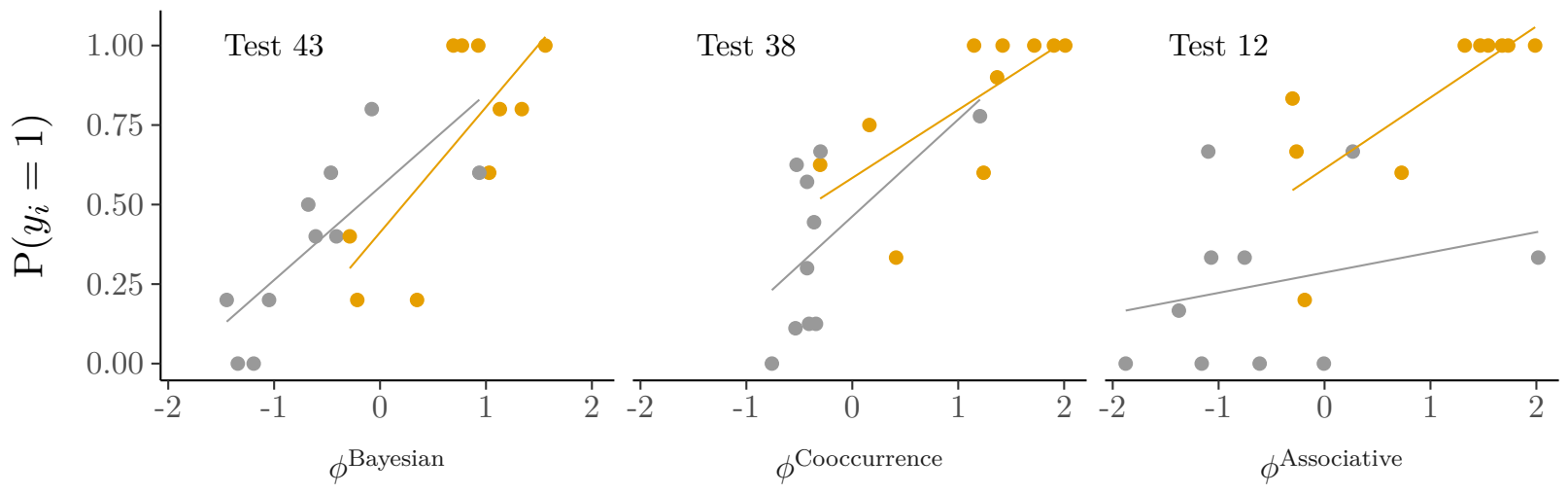

b

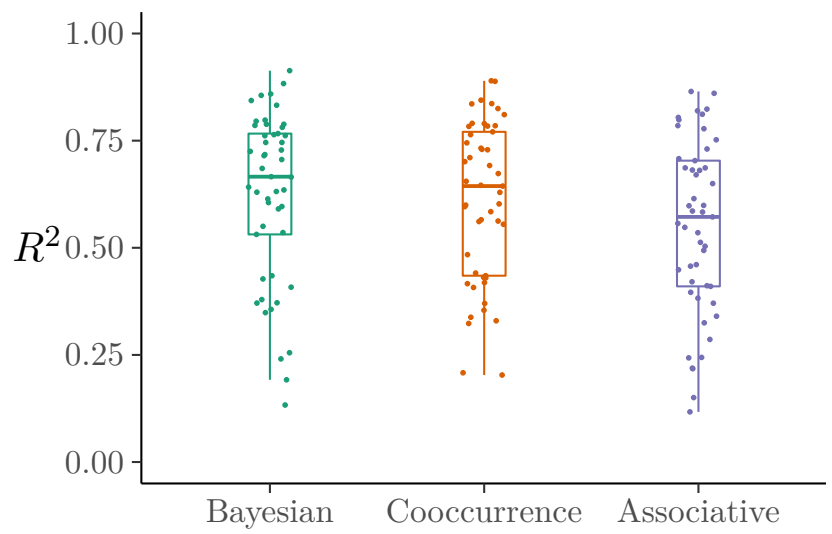

C

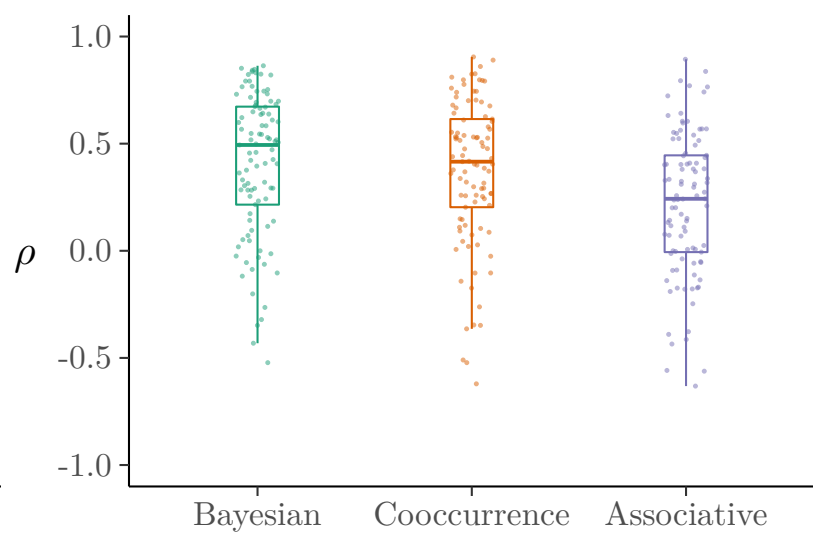

Figure 5. a) Scatterplots of the empirical probability of recognizing each word against the normalized log of the recognition probabilities, denoted by $\phi$, according to each computational model. For each computational model, we chose a representative test (see main text for explanation). In each scatterplot, each point represents a test word, and we distinguish between words that were, and were not, in the text by colour. b) Tukey boxplots showing the distribution of the $R^{2}$ values of the linear regression models predicting, separately for each test, the empirical recognition probabilities as a function of normalized log of the recognition probabilities according to each computational model and whether the word was present or not in the corresponding text. c) Tukey boxplots showing the distribution of Spearman's $\rho$ coefficients between the empirical recognition probabilities and the normalized log of each model's recognition probabilities. In this case, for each test, we calculate $\rho$ separately for the set of test words there were, and were not, in the memory test's text.

for each test, we calculate the empirical probability that each test word therein is recognized, whether veridically or falsely. This is simply the relative frequency with which the participants 
respond that the word is present in the text that they just read. More precisely, the empirical probability of recognizing test word $v$ in memory test $j$ is

$$
q_{v j}=\frac{\sum_{\left\{i: w_{i}=v, t_{i}=j\right\}} y_{i}}{n_{v j}}
$$

where $n_{v j}$ is the total number of trials where the test word is $v$ and the memory test is $j$. In Figure 5a, we provide scatterplots of the relationship between these empirical recognition probabilities and the normalized (i.e., mean $=0$, standard deviation $=1$ ) logarithm of the probability according to each of the three memory models. In each case, we use a different representative (see below for explanation) memory test to construct the scatterplot. Specifically, we use test 43 with the Bayesian model's probabilities, test 38 with the Cooccurrence model, and test 12 with the Associative model. Note that in each scatterplot, there are 20 points, with each one representing a test word in the memory test. Words that had, versus had not, appeared in the corresponding text are distinguished from one another by colour.

Next, separately for each of the three models, and for each of the 50 memory tests, we fit a linear regression model using the empirical recognition probability as the outcome variable and the normalized log of the probability according to the memory model as the predictor, and with a binary covariate that indicates if the word was in fact in the text or not. More precisely, using $\phi_{(j, v)}^{k}$, for $k \in 1,2,3$, to signify the normalized logarithm of $\psi_{(j, v)}^{\text {Bayesian }}, \psi_{(j, v)}^{\text {Cooccurrence }}$, and $\psi_{(j, v)}^{\text {Associative }}$, respectively, and for each memory test $j$, we fit the following linear model,

$q_{v j}^{k}=\beta_{0}+\beta_{1} \phi_{(j, v)}^{k}+\beta_{2} z_{j v}+\epsilon_{j v}, \quad \epsilon_{j v} \sim N\left(0, \sigma^{2}\right), \quad$ for each test word $v$ in memory test $j$,

where $z_{j v}$ signifies if test word $v$ was present in the text corresponding to test $j$. The $R^{2}$ values for all of these regression models are displayed in the Tukey boxplots in Figure 5b. The median $R^{2}$ values for the Bayesian, Cooccurrence, and Associative models are 0.67, 0.64, and 0.57, respectively. These median values occur with test 43 using the Bayesian model's probabilities, test 38 using the Cooccurrence model, and test 12 using the Associative model. Hence our choice of scatterplots in Figure 5a. Clearly, the $R^{2}$ values displayed in Figure 5b give the amount of variation explained in the empirical recognition probabilities by knowing both the probabilities according to each model and also whether the word occurred in each text. In order to isolate the relationship between the empricial recognition probabilities and the models' predictions, for each model and each test, we calculate the Spearman's $\rho$ correlation coefficient separately for the set of 10 words that were, and were not, in the text corresponding to the test. For each model, we display all the values of these Spearman's $\rho$ coefficients in the Tukey boxplots in Figure 5c. The median $\rho$ values for the Bayesian, Cooccurrence, and Associative models are 0.49, 0.42, and 0.24, respectively.

We will now more thoroughly analyse how well the memory models predict the human recognition data. To begin, if any of the models is predictive of the behavioural responses, the $\log$ odds that $y_{i}=1$ should rise as a linear function of the logarithm of the predictor. As such, we can model how well any or all of these predictors predict the behavioural responses using a binary logistic regression with the logarithm of $\psi^{\text {Bayesian }}, \psi^{\text {Cooccurrence }}$, and $\psi^{\text {Associative }}$ as predictors. 
In general, how well any predictor predicts the probability that the participant will respond that $y_{i}=1$ may vary randomly across participants, simply due to individual differences across participants. We refer to these as subject-level random effects. Likewise, how well a predictor predicts the response may vary randomly across different memory tests. For example, the predictors may be better or worse with some texts and not others. We refer to these as text-level random effects. In addition, there should also be word-level random effects given that some individual words may always be more or less memorable than others, regardless of context. Finally, we should also expect that, if $w_{i}$ was in fact present in the preceding text, there should be a higher probability of responding that $y_{i}=1$. This is simply saying that, on average, we should be more likely to correctly recognize words that were in fact in the text than to falsely recognize words that were not there.

Accordingly, we will use the following multilevel binary logistic regression to model how the probability that $y_{i}=1$ varies as a function of the logarithm of the models' predictors:

$\ln \left(\frac{p_{i}}{1-p_{i}}\right)=\beta_{i}^{0}+\sum_{k=1}^{3} \beta_{i}^{k} \phi_{\left(t_{i}, w_{i}\right)}^{k}+z_{i}\left(\beta_{i}^{4}+\sum_{k=1}^{3} \beta_{i}^{4+k} \phi_{\left(t_{i}, w_{i}\right)}^{k}\right)+\zeta_{w_{i}}, \quad$ for each $i \in 1,2 \ldots n$.

Here, $p_{i}$ signifies the probability that $y_{i}=1$. The predictor $z_{i}$ is a binary variable indicating whether $w_{i}$ was present in preceding text. The predictor variables $\phi_{\left(t_{i}, w_{i}\right)}^{1}, \phi_{\left(t_{i}, w_{i}\right)}^{2}, \phi_{\left(t_{i}, w_{i}\right)}^{3}$ are the normalized (i.e., re-scaled to have a mean of zero and standard deviation of 1 over all $i$ ) natural logarithms of $\psi_{\left(t_{i}, w_{i}\right)}^{\text {Bayian }}, \psi_{\left(t_{i}, w_{i}\right)}^{\text {Cooccurrence }}$, and $\psi_{\left(t_{i}, w_{i}\right)}^{\text {Associative }}$, respectively. The coefficients $\beta_{i}^{0}, \beta_{i}^{1} \ldots \beta_{i}^{7}$ are each defined as follows:

$$
\beta_{i}^{l}=\gamma_{s_{i}}^{l}+\xi_{t_{i}}^{l}, \quad \text { for each } l \in 0,1 \ldots 7
$$

Here, $\gamma_{s_{i}}^{l}$ signifies the subject-level random coefficient, and $\xi_{t_{i}}^{l}$ is the text-level random coefficient. These effects are normally distributed. In particular, for each $l \in 0,1 \ldots 7$, and for each subject $s_{i}, \gamma_{s_{i}}^{l} \sim N\left(\mu_{\gamma}^{l}, \tau_{\gamma}^{l}\right)$. Likewise, for each $l \in 0,1 \ldots 7$, and for each text $t_{i}$, $\xi_{t_{i}}^{l} \sim N\left(\mu_{\xi}^{l}, \tau_{\xi}^{l}\right)$. Finally, $\zeta_{w_{i}}$ is a word-level random effect, which is also normally distributed. For each word $w_{i}, \zeta_{w_{i}} \sim N\left(\mu_{\zeta}, \tau_{\zeta}\right)$. Note that this model is a conventional multilevel logistic regression model with fixed and random effects, yet it is presented in a more compact form than usual ${ }^{5}$.

We fit this model to the observed data using Bayesian inference, implemented using the Stan probabilistic programming language (see, e.g., Carpenter et al., 2017) via the R based brms interface (Bürkner, 2017). Specifically, for all unknown variables in the model, which include, for example, the fixed and random effects coefficients, the means and variances of their multilevel distributions, and so on, we compute the posterior distribution over their possible values conditional on the observed data. In other words, on the assumption that

\footnotetext{
${ }^{5}$ Its standard form is clear if we substitute each $\beta_{i}^{l}$ with $\gamma_{s_{i}}^{l}+\xi_{t_{i}}^{l}$, and note also that if any variable $x$ is normally distributed with mean $\mu$ then $x-\mu$ is also normally distributed, but with a zero mean. Proceeding in this manner, it becomes clear, for example, that the fixed effect intercept is $\mu_{\gamma}^{0}+\mu_{\xi}^{0}+\mu_{\zeta}$, the fixed effect for the main effect corresponding to $z_{i}$ is $\mu_{\gamma}^{4}+\mu_{\xi}^{4}$, and so on. Also note that this model is maximal (Barr, Levy, Scheepers, \& Tily, 2013) in the sense that there are subject-level, text-level, and word-level random intercepts and subject-level and text-level random slopes for all predictors.
} 
this model generated the observed data, we obtain the probability distribution over the possible values of all its unknown variables.

Having computed the posterior distribution, we then evaluate the model. We do so by measuring its out-of-sample predictive performance and how this changes when we selectively remove one or more of the three computational memory models' predictor variables. More specifically, we measure out-of-sample predictive performance using Watanabe Akaike Information Criterion (WAIC) (see Appendix $\mathrm{C}$ for more detail about WAIC and the calculation of out-of-sample predictive performance generally). To interpret WAIC values, we first note that, in general, a model's WAIC value is of little meaning in itself, and only becomes meaningful when we compare its value to that of other models that were fit to the same data. The larger the differences in the WAIC scores between two models, the greater the differences in the relative predictive power between the better model, which is the one with the lower value, and the other. While we will avoid the use of any strict thresholds, and especially avoid dichotomizing WAIC differences into significant and non-significant differences, conventional standards (see, for example, Burnham \& Anderson, 2003, Chapter 2 ) hold that WAIC differences of greater than between 4 or 7 indicate clear superiority of the predictive power of the model with the lower WAIC, while differences of 10 or more indicate that the model with the higher value has essentially no predictive power relative to the model with the lower value. More specifically, using the concept of Akaike weights (see Burnham \& Anderson, 2003, p. 75) applied to WAIC values, we can say that if there are just two models being considered, differences in WAIC values equal to 4, 6, 9, or 14 correspond to probabilities of approximately $0.9,0.95,0.99$, and 0.999 , respectively, that the model with the lower WAIC score has the better predictive performance (see also McElreath, 2015, Chapter 6). The standard error of the WAIC difference score should be seen as simply a measure of the uncertainty of the estimation of the difference score, and thus we moderate our interpretation of a WAIC difference by also taking into account its uncertainty.

The WAIC value of the multilevel binary logistic regression presented in Equation 3, is $5,246.72$. Given that our primary focus is to assess how well each of $\psi^{\text {Bayesian }}, \psi^{\text {Cooccurrence }}$, and $\psi^{\text {Associative }}$ predict the behavioural data, we will compare the WAIC of this model, which has all three predictors, to that of the same model but with each predictor separately removed. The results of this comparison are shown in the Table 2a. Focusing initially on just the WAIC differences, we see that compared to when we use all three of the $\psi^{\text {Bayesian }}$, $\psi^{\text {Cooccurrence }}$, and $\psi^{\text {Associative }}$ predictors, removing any one leads to a clear drop, i.e., of at least 7 , in the corresponding model's predictive performance. This tells us that each of the three models improves our prediction of the behavioural data, and also that no one of these models is redundant with respect to the others. However, it is also clear that removing $\psi^{\text {Bayesian }}$ leads to the most substantial drop, i.e., by 24.82 compared to by 10.24 (when $\psi^{\text {Cooccurrence }}$ is dropped) and 7.55 (when $\psi^{\text {Associative }}$ is dropped), in the model's predictive performance. Furthermore, the standard errors of the WAIC differences, relative to the corresponding differences themselves, are large when we drop either $\psi$ Cooccurrence $\left(\Delta_{\mathrm{SE}}^{\mathrm{WAIC}}=7.35, \Delta^{\mathrm{WAIC}}=10.24\right)$ or $\psi^{\text {Associative }}\left(\Delta_{\mathrm{SE}}^{\mathrm{WAIC}}=7.44, \Delta^{\mathrm{WAIC}}=7.55\right)$, compared to when we drop $\psi^{\text {Bayesian }}\left(\Delta_{\mathrm{SE}}^{\mathrm{WAIC}}=11.37, \Delta^{\mathrm{WAIC}}=24.82\right)$.

We may obtain an alternative perspective on these results by comparing a model 


\begin{tabular}{rllll}
\hline & Full model & $\neg \psi^{\text {Bayesian }}$ & $\neg \psi^{\text {Cooccurrence }}$ & $\neg \psi^{\text {Associative }}$ \\
\hline WAIC & 5246.72 & 5271.54 & 5256.96 & 5254.27 \\
$\Delta^{\text {WAIC }}$ & 0.00 & $24.82(11.37)$ & $10.24(7.35)$ & $7.55(7.44)$ \\
\hline
\end{tabular}

a) Full multilevel logistic regression model (i.e. Equation 3), compared to when each predictor is individually dropped.

\begin{tabular}{rlll}
\hline & $\psi^{\text {Bayesian }}, \psi^{\text {Cooccurrence }}$ & $\neg \psi^{\text {Bayesian }}$ & $\neg \psi^{\text {Cooccurrence }}$ \\
\hline WAIC & 5254.27 & 5279.14 & 5263.06 \\
$\Delta^{\text {WAIC }}$ & 0.00 & $24.87(11.52)$ & $8.78(6.27)$
\end{tabular}

b) Multilevel logistic regression model with two predictors, $\psi^{\text {Bayesian }}$ and $\psi^{\text {Cooccurrence }}$, compared to when each predictor is dropped.

\begin{tabular}{rlll}
\hline & $\psi^{\text {Bayesian }}, \psi^{\text {Associative }}$ & $\neg \psi^{\text {Bayesian }}$ & $\neg \psi^{\text {Associative }}$ \\
\hline WAIC & 5256.96 & 5342.82 & 5263.06 \\
$\Delta^{\text {WAIC }}$ & 0.00 & $85.85(19.71)$ & $6.09(6.31)$ \\
\hline
\end{tabular}

c) Multilevel logistic regression model with two predictors, $\psi^{\text {Bayesian }}$ and $\psi^{\text {Associative }}$, compared to when each predictor is dropped.

\begin{tabular}{rllll}
\hline & $\psi^{\text {Bayesian }}$ & $\psi^{\text {Cooccurrence }}$ & $\psi^{\text {Associative }}$ & Null model \\
\hline WAIC & 5263.06 & 5279.14 & 5342.82 & 5369.3 \\
$\Delta^{\text {WAIC }}$ & 0.00 & $16.09(15.81)$ & $79.76(20.86)$ & $106.24(21.65)$ \\
\hline
\end{tabular}

d) Multilevel logistic regression model with $\psi^{\text {Bayesian }}$ predictor alone, compared to with $\psi^{\text {Bayesian }}$ alone, $\psi^{\text {Associative }}$ alone, and a null model with no predictors.

Table 2

Recognition memory experiment model comparison results. Models are compared in terms of their relative out-of-sample predictive peformance, which is measured by differences in their WAIC scores. In each sub-table above, the top row provides, in the first cell on the left, the WAIC value of a model to which others are being compared. The following cells on the first row provide the WAIC values of these models. In the next row of the table, labelled $\Delta^{\mathrm{WAIC}}$, we provide the difference between the WAIC of each model and that of the model to which they are being compared. Also on this row, shown in brackets next to each difference score, we provide the standard error of these differences. 
with two predictors to the model when each one of the two predictors is invidually dropped. Comparing the model with the $\psi^{\text {Bayesian }}$ and $\psi^{\text {Cooccurrence }}$ predictors to the model with each one of these dropped, we have the results shown in Table $2 \mathrm{~b}$. When we drop $\psi^{\text {Bayesian }}$, the loss in predictive power is substantial, with the WAIC increasing by $24.87\left(\Delta_{\mathrm{SE}}^{\mathrm{WAIC}}=11.52\right)$, compared to when we drop $\psi^{\text {Cooccurrence, }}$, in which case the WAIC increases by $8.78\left(\Delta_{\mathrm{SE}}^{\mathrm{WAIC}}=\right.$ 6.27). Similarly, we can compare the model with the $\psi^{\text {Bayesian }}$ and $\psi^{\text {Associative }}$ predictors to the model with each of these predictors individually dropped. This is shown in Table 2c. In this case, when we drop $\psi^{\text {Bayesian }}$, the loss in predictive power is especially large, with the WAIC increasing by $85.85\left(\Delta_{\mathrm{SE}}^{\mathrm{WAIC}}=19.71\right)$, compared to when we drop $\psi^{\text {Associative }}$, whereby the WAIC increases by $6.09\left(\Delta_{\mathrm{SE}}^{\mathrm{WAIC}}=6.31\right)$.

Finally, we may directly compare the WAIC score of the model based on $\psi^{\text {Bayesian }}$ alone to that the model using either $\psi^{\text {Cooccurrence }}$ alone, $\psi^{\text {Associative }}$ alone, or a null model that drops all three predictors ${ }^{6}$, as shown in Table $2 \mathrm{~d}$. Here, we can see that relative to the predictive performance of the $\psi^{\text {Bayesian }}$ predictor, the $\psi^{\text {Associative }}$ predictor has clearly poorer predictive performance $\left(\Delta^{\mathrm{WAIC}}=79.76, \Delta_{\mathrm{SE}}^{\mathrm{WAIC}}=20.86\right)$. The difference in the WAIC between $\psi^{\text {Bayesian }}$ and $\psi^{\text {Cooccurrence }}$ is also large $\left(\Delta^{\text {WAIC }}=16.09\right)$, but also with a relatively large standard error $\left(\Delta_{\mathrm{SE}}^{\mathrm{WAIC}}=15.81\right)$.

Recall memory data analyses. We now turn to the recall memory tests. In each recall memory test, the participant was asked to list as many words as they could remember from the text that they had just read. After removing any non-word responses, the average number of words that were recalled in each test was 7.18 . On average, $31 \%$ of these were false recalls, i.e., words that did not occur in the text (over $83 \%$ of the 276 recall tests have false recall rates of greater than $10 \%)$.

As in the case of the recognition test data, the primary aim of our analysis of the recall test data is to evaluate how well each of our three computational models of memory predict the memory test responses. Unlike in the case of the recognition tests, however, where each response was binary, each response in a recall test, being a word in the English language, is a categorical variable with as many values as there are words in the vocabulary. We will label these possible values by the integers $1 \ldots V$, where $V=49328$ is the number of words in the vocabulary ${ }^{7}$. As such, our entire set of recall data response variables, i.e., all the responses produced in all recall tests across all participants, can be labelled $y_{1}, y_{2} \ldots y_{n}$, where each $y_{i} \in 1 \ldots V$. As with the recognition data, for each trial $i$, we again

${ }^{6}$ The null model has the following form:

$$
\ln \left(\frac{p_{i}}{1-p_{i}}\right)=\beta_{i}^{0}+z_{i} \beta_{i}^{4}+\zeta_{w_{i}}, \quad \text { for each } i \in 1,2 \ldots n,
$$

where all terms have the same meaning as in Equation 3. In other words, the null model is identical to full model but with the $\phi^{1}, \phi^{2}$, and $\phi^{3}$ predictors dropped. Crucially, it models the log odds of recognition as a linear function of whether the word was in the memory test's text, and also allows for random variation in this relationship across both subjects and tests. In other words, it is a non-trivial null model that, when compared to a model with either one of the $\phi^{1}, \phi^{2}$, and $\phi^{3}$ predictors, can be used to isolate their respective effects.

${ }^{7}$ The computational memory models that we are evaluating all have a vocabulary of exactly $V=49328$ words. Any responses by participants in the recall tests that were outside this vocabulary set were not considered. 
a

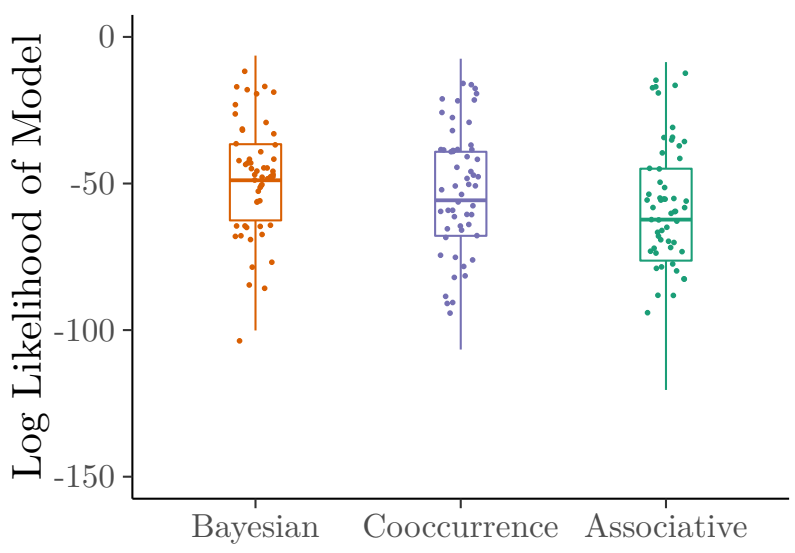

b

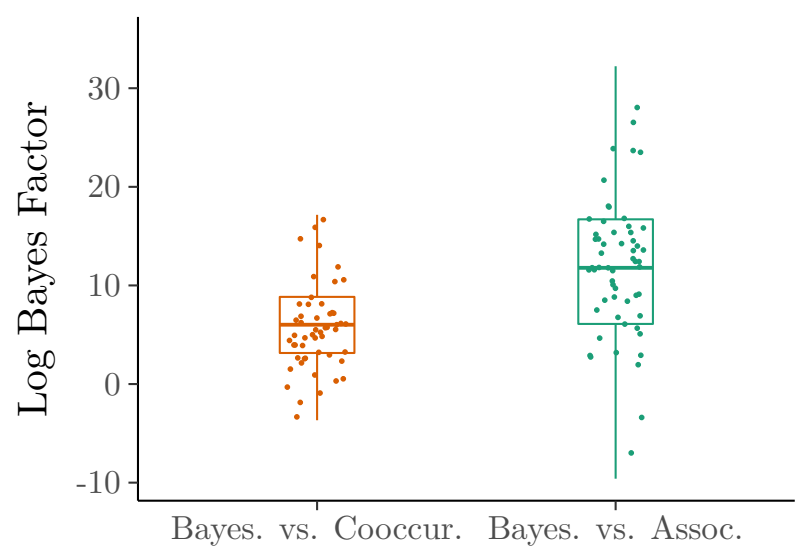

Figure 6. a) Tukey boxplots of something. b) Tukey boxplots showing the distribution of the $R^{2}$ values of the linear regression models predicting, separately for each test, the empirical recognition probabilities as a function of normalized log of the recognition probabilities according to each computational model and whether the word was present or not in the corresponding text.

also have $s_{i} \in 1 \ldots 216$ and $t_{i} \in 1 \ldots 50$, which identify the participant and the memory test, respectively, corresponding to the $i$ th observation. Each response $y_{i}$ can be viewed as a sample from participant $s_{i}$ 's subjective probability distribution of the words in the text corresponding to test $t_{i}$. This subjective probability distribution essentially represents participant $s_{i}$ 's memory of the words in the text corresponding to test $t_{i}$. In other words, having read the text in test $t_{i}$, participant $s_{i}$ 's memory of what words were and were not present in the text can be described as a probability distribution over the entire vocabulary of words, presumably with most words being assigned a trivial or negligible probability and with most of the probability mass being assigned to a relatively small number of words. The participants' subsequent recall responses can then be viewed as samples from this probability distribution.

For any given recall test, the likelihood, or its logarithm, of the responses according to the Bayesian, Cooccurrence, or Associative models are straightforward to calculate. For example, for subject $j$ performing test $k$, the log of the likelihood of the observed responses according to the Bayesian model is

$$
L_{j k}^{\text {Bayesian }}=\sum_{\left\{i: s_{i}=j, t_{i}=k\right\}} \log \left(\psi_{\left(k, y_{i}\right)}^{\text {Bayesian }}\right) .
$$

We may define $L_{j k}^{\text {Cooccurrence }}$ and $L_{j k}^{\text {Cooccurrence }}$ identically using $\psi^{\text {Cooccurrence }}$ and $\psi^{\text {Associative }}$, respectively, in place of $\psi^{\text {Bayesian}}$. In Figure $6 \mathrm{a}$, we display the distribution the $\log$ of the likelihood of the responses in all 276 recall memory tests, according the Bayesian, Cooccurrence, or Associative models. It is clear from this figure that the Bayesian model has a likelihood across all tests on average (median log likelihood, -48.93) that is higher 
than the Cooccurrence model (median log likelihood, -56.07) and the Associative model (median log likelihood, -62.46). In addition, note that from a Bayesian model comparison perspective, for any $j$ and $k$, for example,

$$
L_{j k}^{\text {Bayesian }}-L_{j k}^{\text {Cooccurrence }}
$$

is the log of the Bayes factor of the Bayesian model compared to the Cooccurrence model, with the log Bayes factor for any other pair of models being analogously defined. In Figure 6b, we display the distributions of the log Bayes factors comparing the Bayesian to the Cooccurrence model, and the Bayesian to the Associative model, across all 276 tests. The log of the Bayes factor comparing the Bayesian model to the Cooccurrence model is greater than zero (hence the Bayes factor itself is greater than 1) in $93.10 \%$ of cases. Likewise, the $\log$ of the Bayes factor comparing the Bayesian model to the Associative model is greater than zero in $92.80 \%$ of cases. Furthermore, according to one of the widely held standards for interpreting Bayes factors (Kass \& Raftery, 1995), a Bayes factor of 3, and so a log Bayes factor of $\log (3)$, when comparing a model $M_{1}$ to another model $M_{0}$ indicates clear or unambiguous evidence in favour of the model $M_{1}$. By this standard, the Bayesian model is clearly superior to the Cooccurrence model in $89.10 \%$ of cases, and clearly superior to the Associative model in $91.70 \%$ of cases. These preliminary results therefore show that even at the level of each individual recall test done by each individual participant, the Bayesian model is superior to the Cooccurrence and Associative model in the vast majority of cases.

We may also compare the models using the data from all tests combined. The log of the likelihood of all the observed responses, across all tests and all participants, according to the Bayesian model is

$$
L^{\text {Bayesian }}=\sum_{i=1}^{n} \log \left(\psi_{\left(t_{i}, y_{i}\right)}^{\text {Bayesian }}\right),
$$

with $L^{\text {Cooccurrence }}$ and $L^{\text {Cooccurrence }}$ defined identically using $\psi^{\text {Cooccurrence }}$ and $\psi^{\text {Associative }}$, respectively. The log of the Bayes factor comparing the Bayesian model to the Cooccurrence model based on all the data is $L^{\text {Bayesian }}-L^{\text {Cooccurrence }}=1,748.34$, and the corresponding $\log$ of the Bayes factor comparing the Bayesian model to the Associative model is $L^{\text {Bayesian }}$ $L^{\text {Associative }}=3,420.73$. Clearly, these results indicate an overwhelming case in favour of the Bayesian model over either the Coocurrence and Associative model.

As with the analysis of the recognition memory data, for the case of the recall memory data, our primary aim is to analyse how well each of the three computational memory models predict the observed responses. We will model the relationship between these three predictors and the recall responses using multilevel categorical logistic regression. In particular, for each $i \in 1 \ldots n$, we will model the recall response $y_{i}$ as a categorical random variable whose probability distribution is $\pi_{i}$ (i.e., $\pi_{i}$ is an array of length $V$, and for all $v \in 1 \ldots V, 0 \leq \pi_{i v} \leq 1$ and $\left.\sum_{v=1}^{V} \pi_{i v}=1\right)$, and model $\pi_{i}$ as a function of the three predictors. Specifically, our multilevel categorical regression model is as follows: for all $i \in 1 \ldots n, y_{i} \sim \operatorname{Categorical}\left(\pi_{i}\right)$, and for all $v \in 1 \ldots V$,

$$
\pi_{i v}=\frac{e^{\sum_{k=1}^{3} \beta_{i}^{k} \phi_{\left(t_{i}, v\right)}^{k}}}{\sum_{v=1}^{V} e^{\sum_{k=1}^{3} \beta_{i}^{k} \phi_{\left(t_{i}, v\right)}^{k}} .}
$$


The coefficients $\beta_{i}^{1}, \beta_{i}^{2}, \beta_{i}^{3}$ are each defined as

$$
\beta_{i}^{l}=\gamma_{s_{i}}^{l}+\xi_{t_{i}}^{l}, \quad \text { for each } l \in 1,2,3,
$$

with $\gamma_{s_{i}}^{l}$ being the subject-level random coefficient, and $\xi_{s_{i}}^{l}$ being the text-level random coefficient. These effects are normally distributed as follows: For each $l \in 1,2,3$, for each subject $s_{i}, \gamma_{s_{i}}^{l} \sim N\left(\mu_{\gamma}^{l}, \tau_{\gamma}^{l}\right)$, and for each text $t_{i}, \xi_{t_{i}}^{l} \sim N\left(\mu_{\xi}^{l}, \tau_{\xi}^{l}\right)$.

In Table $3 \mathrm{a}$, we see that compared to when we use all three of the $\psi^{\text {Bayesian }}, \psi^{\text {Cooccurrence }}$, and $\psi^{\text {Associative }}$ predictors, removing any one leads to a clear drop in the categorical logistic regression's predictive performance. In particular, there is a rise of at least 69.61 in the models' WAIC scores if any one of these three predictors is dropped, and in all cases there are relatively very small standard errors on these differences. However, it is also evident that the rise in the WAIC score when $\psi^{\text {Bayesian }}$ is dropped, i.e. 3,364.06, is considerably larger than when either $\psi^{\text {Cooccurrence }}$ or $\psi^{\text {Associative }}$ is dropped, which lead to rises in WAIC scores of 787.44 , and 69.61 , respectively.

We may also compare the predictive performance of the model using just $\psi^{\text {Bayesian }}$ and $\psi$ Cooccurrence to that of the model when either of these predictors is dropped. In this case, we see when $\psi^{\text {Bayesian }}$ is dropped, there is a WAIC rise in 3,518.25, By contrast, when $\psi^{\text {Cooccurrence }}$ is dropped, the rise in WAIC, i.e., 1,046.08, is considerably less. Similarly, we may compare the predictive performance of the model using just $\psi^{\text {Bayesian }}$ and $\psi^{\text {Associative }}$ to that of the model when either is dropped. When $\psi^{\text {Bayesian }}$ is dropped, there is a WAIC rise of $3,256.84$, and when $\psi^{\text {Associative }}$ is dropped, there is a WAIC rise of 328.25.

Finally, we can compare the WAIC of the models using either $\psi^{\text {Bayesian }}, \psi^{\text {Cooccurrence }}$, or $\psi^{\text {Associative }}$, alone. The WAIC values of these models are displayed in the top row of Table $3 \mathrm{~d}$. In addition, we also provide the WAIC value of a null model with no predictors. On the second row of that table, we show the rise in the WAIC value of each model compared to that of the $\psi^{\text {Bayesian }}$ model. From this, it is clearly evident that the $\psi^{\text {Bayesian }}$ model has a remarkably lower WAIC value than the other models, being 2,472.17 lower than that of the nearest model.

\section{Results Summary}

From exploratory analyses of both the recognition and recall data, across all texts used in this experiment, the probability of recognizing or recalling any given word, whether falsely of veridically, is well predicted by the conditional probabilities of each word according to the Bayesian, Cooccurrence, and Associative computational models. However, in both the recognition and the recall tests, and in both the exploratory analyses and the eventual multilevel logistic regression analyses, there is consistently strong, or even overwhelming, evidence in favour of the Bayesian model being the best predictor of the probability of recognizing or recalling any given word in a text just read. For example, in the case of the logistic regression analyses of the recognition results, dropping the Bayesian model's predictions, but not those of the Cooccurrence or Associative models, from the regression models leads to substantial drop the out-of-sample predictive performance as measured by WAIC. In the case of the logistic regression analyses of the recall memory tests, while dropping any one of the three computational models' predictions leads to a substantial 


\begin{tabular}{lllll}
\hline & Full model & $\neg \psi^{\text {Bayesian }}$ & $\neg \psi^{\text {Cooccurrence }}$ & $\neg \psi^{\text {Associative }}$ \\
\hline WAIC & 22793.29 & 26157.35 & 23580.73 & 22862.90 \\
$\Delta^{\text {WAIC }}$ & 0 & $3364.06(108.39)$ & $787.44(51.04)$ & $69.61(16.28)$ \\
\hline
\end{tabular}

a) Full multilevel categorical logistic regression model (i.e. Equation 4), compared to when each predictor is individually dropped.

\begin{tabular}{rlll}
\hline & $\psi^{\text {Bayesian }}, \psi^{\text {Cooccurrence }}$ & $\neg \psi^{\text {Bayesian }}$ & $\neg \psi^{\text {Cooccurrence }}$ \\
\hline WAIC & 22862.90 & 26381.15 & 23908.98 \\
$\Delta^{\text {WAIC }}$ & 0 & $3518.25(108.86)$ & $1046.08(50.71)$ \\
\hline
\end{tabular}

b) Multilevel categorical logistic regression model with two predictors, $\psi^{\text {Bayesian }}$ and $\psi^{\text {Cooccurrence }}$, compared to when each predictor is dropped.

\begin{tabular}{rlll}
\hline & $\psi^{\text {Bayesian }}, \psi^{\text {Associative }}$ & $\neg \psi^{\text {Bayesian }}$ & $\neg \psi^{\text {Associative }}$ \\
\hline WAIC & 23580.73 & 26837.57 & 23908.98 \\
$\Delta^{\text {WAIC }}$ & 0 & $3256.84(119.86)$ & $328.25(37.80)$ \\
\hline
\end{tabular}

c) Multilevel categorical logistic regression model with two predictors, $\psi^{\text {Bayesian }}$ and $\psi^{\text {Associative }}$, compared to when each predictor is dropped.

\begin{tabular}{rllll}
\hline & $\psi^{\text {Bayesian }}$ & $\psi^{\text {Cooccurrence }}$ & $\psi^{\text {Associative }}$ & Null model \\
\hline WAIC & 23908.98 & 26381.15 & 26837.57 & 27742.54 \\
$\Delta^{\text {WAIC }}$ & 0 & $2472.17(64.38)$ & $2928.59(91.84)$ & $3833.56(102.58)$ \\
\hline
\end{tabular}

d) Multilevel categorical logistic regression model with $\psi^{\text {Bayesian }}$ predictor alone, compared to with $\psi^{\text {Cooccurrence }}$ alone, $\psi^{\text {Associative }}$ alone, and a null model with no predictors.

Table 3

Recall memory experiment model comparison results. As was done for the recognition memory experiment analysis, models are compared in terms of their relative out-of-sample predictive peformance, which is measured by differences in their WAIC scores. This table is laid out identically to Table 2: In each sub-table, the first cell in the top row provides the WAIC value of a model to which others are being compared. The following cells provide the WAIC values of these models. In the next row of the table, the difference between the WAIC of each model and that of the model to which they are being compared is provided. In brackets next to each difference score, we provide the standard error of these differences. 
drop in out-of-sample predictive performance, dropping the Bayesian model leads to an overwhelmingly larger drop than dropping those of the Coocurrence and Associative models. Likewise, the WAIC score of the regression model using the predictions of the Bayesian model alone is overwhelmingly better than those using the predictions of either the Coocurrence and Associative models.

\section{Discussion}

Understanding human memory for spoken and written language that goes beyond isolated words or sentences informs our understanding of human memory generally and also language comprehension. It has been the subject of investigation in psychology for almost a century and since the seminal work on this topic, but especially since the advent of schema theory based accounts beginning in the 1970s, there has been something close to a consensus on the broad and general characteristics of how human memory for text works. Roughly speaking, according to this account, the recognition or recall of the content of a text is based on querying a representation of that text that is constructed on the basis of background knowledge and experience. Despite the widespread appeal of this account, and despite there being ample empirical evidence showing that we use our background knowledge to make inferences and associations concerning text content and that these inferences then influence our memory, this account is largely an informal theory. As such, it does not lead to precise and testable predictions, is challenging to falsify, or to elaborate as a theory of memory or language comprehension. The purpose of this paper, therefore, is to provide a formal or computational version of this widely held account of text memory. In particular, we describe human memory for text as a Bayesian computational model whereby background knowledge, in the form of knowledge of coarse grained statistical patterns across spoken and written language, is used via Bayes's rule to infer a semantic representation of a text's content, and then this is used via posterior predictive inference as the basis of our memory of what words were and were not present in text. We have implemented this account as a HDPTM, trained it on an approximately 80 million word portion of the BNC, and evaluated it using recognition and recall memory results from behavioural experiments involving over 200 participants.

The behavioural experiments show that after reading everyday texts under normal reading conditions, participants nonetheless have relatively high false positive and false negative recognition and recall rates. In other words, when we read everyday texts, we have a relatively high probability of falsely remembering items that were not in the text, and also missing items that were there. The probability of remembering or not remembering any given item in the text, whether falsely or veridically, is well predicted by the Bayesian model. For example, the median $R^{2}$ value of linear models predicting recognition memory rates from the posterior predictive probabilities of the Bayesian model is 0.67 . The main focus of our evaluation analyses, however, is comparing the predictions of the Bayesian model against those of nontrivial alternative models. We specifically compared the Bayesian model to two association based models of text memory. According to both of these accounts, which words we do an do not remember from a text, whether falsely or veridically, is based on the average associative strength between the words in the text and any other word (see Equation 1, Page 17; Equation 2, Page 18). For example, if word $w_{j^{\prime} i^{\prime}}$ is highly associated on average with the words in text $t_{j^{\prime}}$, then it has a high probability of being remembered after having read 
that text. Compared to these associative models of text memory, by every measure used in the analyses, the predictions of the Bayesian model are superior, often overwhelmingly so. We take this collection of results as strong evidence in favour of the Bayesian account of text memory that we have presented in this paper.

Before we attempt to draw more general conclusions from this work, we will discuss a number of noteworthy issues that arose from the analyses, and also address some potential criticisms of how we have interpreted these analyses. To begin, it is noteworthy that there is considerably stronger evidence in favour of the Bayesian model from the results of the recall memory tests than from those of the recognition memory tests. Although in both cases the Bayesian model is shown to be preferrable to the alternatives, the superiority of the Bayesian is remarkably stronger based on the recall memory results. One possible explanation of this is that recall memory tests are a more sensitive measure of knowledge effects on text memory (Long, Prat, Johns, Morris, \& Jonathan, 2008), which is precisely what the Bayesian model is designed to describe. According to this account, recognition memory only minimally involves the semantic representation of the text's content, and the acceptance or rejection of the words in the recognition test can be accomplished using a more superficial representation of the text. While this is an interesting and practically important possibility, an alternative is that the recognition tests used here were not optimally designed to discriminate between the predictions of the Bayesian and the associative models. The recognition test word lists were designed by choosing keywords from the text itself and from its adjacent texts. This method gave us an objective means to create lists where all words were likely to be related to the text's content (with half of the words actually occurring in the text and the others not), and to be theory neutral in that it did not rely on assumptions of any particular theory. However, it would also be possible to objectively design lists that can maximally discriminate between the predictions of the Bayesian models and its alternatives. Roughly speaking, a procedure such as this applied to the case of discriminating between two alternative models, would create lists that contained mixtures of words, some of which were highly predicted by the first model and not the second, while others were highly predicted by the second and not the first. Lists designed in this manner would not a priori favour one model over the other but would be able to maximize the ability to discriminate between their respective predictions. We consider this kind of optimal experimental design as an important option in future research on this topic of theoretical accounts of text memory.

Another noteworthy finding from the analyses was that although what we have termed the Cooccurrence and Associative models are both in fact associative models of text memory, the Coccurrence model had consistently better performance than the Associative model. Recall that these two models differ from one another solely in terms of their definition of the association between two words, with the Coccurrence model defining association in terms of statistical cooccurrence within a corpus, and the Associative model defining association in terms of word association norm data. One relatively simple explanation for the difference in the predictive accuracy of these two models is simply a matter of sparse or missing data. Although the association norms that we have used is a very large example of its kind, it nonetheless only provides associates for a set of approximately 10,000 word types. Moreover, obtaining reliable data for low probability associates of any given word generally requires prohibitively large numbers of responses. We endeavoured to minimize the effect of data 
sparsity arising from the word association norms by only using texts where most words were in the word association norm stimulus set. Nonetheless, this may not be sufficient to make the predictions based on these norms comparable to those based on the cooccurrence statistics, which are obtained from a relatively large corpus of 80 million word tokens and around 50,000 word types. If this explanation of the difference is correct, there may be nothing theoretically interesting about the relative performance of the Cooccurrence and Associative models.

Although we have concluded that the Bayesian model is empirically supported by behavioural data, and is supported to a greater extent than some suitable and nontrivial alternative models, it may nonetheless still be questioned whether different alternative models ought to be considered. In particular, it may be argued that some models not tested here may better account for the behavioural data. We can take it for granted that there are an unlimited number of alternative models that we can consider, and that there are undoubtedly in principle other models that better predict the behavioural data than any of those considered here. However, the objective of this paper is not to find a computational model that best predicts recognition and recall memory of everyday texts. Rather, the objective is primarily to show that a widely held general account of memory for text can be described as a Bayesian computational model, and that this model can make precise testable predictions that are then subsequently supported by behavioural evidence. The associative models to which we compare the Bayesian model are chosen primarily to provide a context by which we can interpret the predictions of the Bayesian model, and were chosen because they are theoretically plausible but yet still theoretically distinct from the the Bayesian model. We also consider the versions of the Bayesian model and the associative models that were used here to be minimal models of their kinds. In other words, more complex versions of both the Bayesian and the associative models are possible and justifiable. For example, an obvious major simplication in the Bayesian model is that it is a bag of words model. Likewise, there are many possible variants and extensions of the spreading activation in the associative network that is assumed by the associative models that we have used here. Developing and testing these more complex variants, as well as considering other theoretical alternatives, will be the subject of future work.

Turning to some more general implications of this work, we may begin by considering the issue of false recognition and recall in memories for text. The DRM effect of Roediger and McDermott (1995) shows us that spontaneous false recall and recognition of words can occur at high rates after reading word lists. We will take it for granted here that this effect is robust, reliable, generalizable, and is due to genuine false memories as opposed to demand characteristics of the experiment or a liberal response bias (see Gallo, 2010, 2006, for discussion). However, the word lists in the DRM task were all specially constructed stimuli. In the present work, we show that when reading randomly selected texts from a representative corpus of English, false memories can still occur at relatively high rates: in our experiment, the false positive recognition rate was $28 \%$ and the false positive recall rate was $31 \%$. Crucially, these are not random errors. Falsely remembered words are words that, though they were not in fact present in the text, are highly predicted on the basis of the semantic representation of the text. This finding underlines two important points. First, false memories are exceptionally commonplace, occurring with high probability with 
almost everything we read or hear (as mentioned above, over $92 \%$ of the experiment's 289 separate recognition tests and over $83 \%$ of the experiment's 276 separate recall tests lead to false memory rates of greater than 10\%). Second, false memories of this kind are a natural by-product of an otherwise adaptive and well designed memory system, one that is based on a semantic representation of what we experience that is built up from our background knowledge. Put another way, the work presented here implies that that human memory should be seen not as a type of recording and play-back system, albeit a noisy one, but more like a probabilistic inference based system. We infer a representation of the meaning of what we experience, and our memories are essentially an inference of which items are and are not compatible with that representation. Viewed from this perspective, false memories are an inevitable consequence of this inferential system.

An additional implication of this work is that it questions the validity of associationist accounts of episodic memory. Associationism has a long and venerable history in psychology, being prominent in Artistotle's accounts of learning and memory (see Sorabji, 2004), an integral part of the empiricist philosophies of Locke, Hume, Berkeley, Hartley and others (see Hearnshaw, 1987), and arguably a central feature of modern connectionist models of cognition (see, for example, Rumelhart \& McClelland, 1987; Elman et al., 1996; McClelland et al., 2010). An associationist account of episodic memory, roughly speaking, posits that observed stimuli activate representations, which in turn activate associated representations through a process of spreading activation (see, for example, Anderson, 1983; Neely, 1977), and the eventual distribution of activation is the memory representation. This general account is essentially being tested by both of the associative models that we have used here. While both models, and especially the Cooccurrence model (for reasons discussed above), have empirical support from the recognition and recall data, there is consistently less empirical support for these models than for the Bayesian model. From these findings, we may speculate that although the associationist and spreading activation account of episodic memory is the dominant account of the DRM effect (see, for example, Roediger et al., 2001; Gallo, 2006), the DRM effect may be explained as well, if not better, by a Bayesian account of word list memory that is similar to the Bayesian text memory model described here. We consider this to be an important hypothesis for future investigation. In addition, our results support an account of episodic memory that is "based on meaning, not on meaningless associations" (Reyna et al., 2016, p. p4). As we explained, our Bayesian model is a computational version of a more general account of memory that holds that recall and recognition are based on the semantic representation of what is experienced. In other words, a vital feature of this more general account is that memories depend on meaningful representations. In the Bayesian model, this representation of the meaning is a representation of the discourse topics in the text, each exemplified by clusters of inter-related words. By contrast, associationist accounts are based on physical or mechanistic network models that make no commitment to the associations in the network (i.e., edges between vertices) being based on meaning, and in fact these are generally assumed to be primarily based on physical or temporal contiguity. Given that the greater degree of empirical support for the Bayesian model, we can take our results as favouring accounts of episodic memory that are based on representations of meaning rather than accounts based on physical or mechanistic models of activity spreading in a network. 
A final implication, and one that follows from the point just discussed, is that this work shows the value of rational accounts (Anderson, 1990; Chater \& Oaksford, 1999; Marr, 1982) of memory. In general, rational models of cognition, which are usually considered to be coextensive with Bayesian models of cognition, consider the computational problems that are being faced by, for example, vision (Kersten, Mamassian, \& Yuille, 2004), categorization (Sanborn, Griffiths, \& Navarro, 2010), reasoning (Oaksford \& Chater, 1994), and then consider normative solutions to these problems. Anderson (1990), Anderson (1989), Anderson and Schooler (1991) describe the problem faced by human memory, uncontroversially, as the storage of information that may be relevant, for some task or other, at some point in the future. When applied to memory for text, determining what may be relevant in what we are reading or hearing crucially requires identifying major discourse topics in the text. These topics are effectively coarse grained statistical patterns that are learned through experience with spoken and written language, and whose presence can then be then inferred in any given text. Having outlined the nature of the problem in these probabilistic or statistical terms, a normative solution in terms of Bayesian inference follows naturally. While valid criticisms of this rational models approach have been raised (Bowers \& Davis, 2012; Jones \& Love, 2011), we nonetheless view it as a principled way of generating formal hypotheses concerning aspects of cognition that may then be evaluated empirically. This is how we aimed to use this approach here, and see the real value of the rational approach to text memory being the extent to which it has allowed us to make precise and testable predictions about what will and will not be remembered, whether falsely or veridically, from what we read and hear. 


\section{References}

Abbott, V., Black, J. B., \& Smith, E. E. (1985). The representation of scripts in memory. Journal of Memory and Language, 24 (2), 179-199.

Alba, J., \& Hasher, L. (1983). Is Memory Schematic? Psychological Bulletin, 93(2), 203-231.

Anderson, J. R. (1983). A spreading activation theory of memory. Journal of Verbal Learning and Verbal Behavior, 22(3), 261-295.

Anderson, J. R. (1989). A rational analysis of human memory. In H. I. Roediger, E. Tulving, H. L. Roediger, \& F. I. M. Craik (Eds.), Varieties of memory and consciousness: Essays in honour of endel tulving (pp. 195-210). L. Erlbaum Associates.

Anderson, J. R. (1990). The Adaptive Character of Thought. Hillsdale, NJ: Erlbaum.

Anderson, J. R., \& Schooler, L. J. (1991). Reflections of the environment in memory. Psychological Science, 2(6), 396-408.

Andrews, M. (2019a). A Gibbs Sampler for a Hierarchical Dirichlet Process Mixture Model. PsyArXiv. doi:10.31234/osf.io/ebzt8

Andrews, M. (2019b). Gustav: A probabilistic topic modelling toolbox. Zenodo. doi:10.5281/zenodo.51824

Andrews, M. (2019c). Wilhelm: A web application framework for online behavioural experiments. Zenodo. doi:10.5281/zenodo.2647481

Barclay, J. R. (1973). The role of comprehension in remembering sentences. Cognitive Psychology, 4(2), 229-254.

Barr, D. J., Levy, R., Scheepers, C., \& Tily, H. J. (2013). Random effects structure for confirmatory hypothesis testing: Keep it maximal. Journal of Memory and Language, $68(3), 255-278$.

Bartlett, F. C. (1932). Remembering: A Study in Experimental and Social Psychology. Cambridge: Cambridge University Press.

Blei, D. M. (2012). Probabilistic topic models. Communications of the ACM, 55(4), 77-84.

Blei, D., Ng, A., \& Jordan, M. (2003). Latent dirichlet allocation. Journal of Machine Learning Research, 3, 993-1022.

Bower, G., Black, J., \& Turner, T. (1979). Scripts in Memory for Text. Cognitive Psychology, 11(2), 177-220.

Bowers, J. S., \& Davis, C. J. (2012). Bayesian just-so stories in psychology and neuroscience. Psychological Bulletin, 138(3), 389.

Brainerd, C., \& Mojardin, A. (1998). Children's and adults' spontaneous false memories: Long-term persistence and mere-testing effects. Child Development, 69(5), 13611377 . 
Brainerd, C., Reyna, V., \& Estrada, S. (2006). Recollection rejection of false narrative statements. Memory, $14(6), 672-691$.

Bransford, J. D., Barclay, J. R., \& Franks, J. J. (1972). Sentence memory: A constructive versus interpretive approach. Cognitive Psychology, 3(2), 193-209.

Bransford, J. D., \& Franks, J. J. (1971). The abstraction of linguistic ideas. Cognitive Psychology, 2(4), 331-350.

Bransford, J. D., \& Johnson, M. K. (1972). Contextual prerequisites for understanding: Some investigations of comprehension and recall. Journal of Verbal Learning and Verbal Behavior, 11(6), 717-726.

British National Corpus Consortium. (2007). The British National Corpus, version 3 (BNC XML Edition). Distributed by Bodleian Libraries, University of Oxford, on behalf of the BNC Consortium. Oxford University Computing Services: http://www.natcorp.ox.ac.uk/.

Bürkner, P.-C. (2017). brms: An R package for Bayesian multilevel models using Stan. Journal of Statistical Software, 80(1), 1-28. doi:10.18637/jss.v080.i01

Burnham, K. P., \& Anderson, D. R. (2003). Model selection and multimodel inference: A practical information-theoretic approach. Springer Science \& Business Media.

Cain, K., Oakhill, J. V., Barnes, M. A., \& Bryant, P. E. (2001). Comprehension skill, inference-making ability, and their relation to knowledge. Memory $\mathscr{E}$ Cognition, $29(6), 850-859$.

Carpenter, B., Gelman, A., Hoffman, M., Lee, D., Goodrich, B., Betancourt, M., ... Riddell, A. (2017). Stan: A Probabilistic Programming Language. Journal of Statistical Software, $76(1)$.

Chater, N., \& Oaksford, M. (1999). Ten years of the rational analysis of cognition. Trends Cognitive Science, 3(2), 57-65.

Connor, R. J., \& Mosimann, J. E. (1969). Concepts of independence for proportions with a generalization of the dirichlet distribution. Journal of the American Statistical Association, 64(325), 194-206.

Cook, A. E., Limber, J. E., \& O'Brien, E. J. (2001). Situation-based context and the availability of predictive inferences. Journal of Memory and Language, 44 (2), 220234 .

Crocker, M. W., Knoeferle, P., \& Mayberry, M. R. (2010). Situated sentence processing: The coordinated interplay account and a neurobehavioral model. Brain and Language, 112(3), 189-201.

Danvers, A. F., \& Shiota, M. N. (2017). Going off script: Effects of awe on memory for script-typical and-irrelevant narrative detail. Emotion, 17(6), 938.

De Deyne, S., \& Storms, G. (2017). Small world of words, www.smallworldofwords.org. 
Dewhurst, S. A., Pursglove, R. C., \& Lewis, C. (2007). Story contexts increase susceptibility to the drm illusion in 5-year-olds. Developmental Science, 10(3), 374-378.

Elman, J., Bates, E., Johnson, M., Karmiloff-Smith, A., Parisi, D., \& Plunkett, K. (1996). Rethinking innateness: Connectionism in a developmental framework. Cambridge, MA: MIT Press.

Ferguson, T. S. (1973). A bayesian analysis of some nonparametric problems. The Annals of Statistics, 209-230.

Frank, S. L., Koppen, M., Noordman, L. G., \& Vonk, W. (2003). Modeling knowledge-based inferences in story comprehension. Cognitive Science, 27(6), 875-910.

Frank, S. L., Koppen, M., Noordman, L. G., \& Vonk, W. (2008). World knowledge in computational models of discourse comprehension. Discourse Processes, 45(6), 429-463.

Gallo, D. (2006). Associative illusions of memory: False memory research in DRM and related tasks. Psychology Press.

Gallo, D. A. (2010). False memories and fantastic beliefs: 15 years of the drm illusion. Memory 8 Cognition, 38(7), 833-848.

Garnham, A. (1981). Mental models as representations of text. Memory $\&$ Cognition, 9(6), $560-565$.

Ghosh, V. E., \& Gilboa, A. (2014). What is a memory schema? A historical perspective on current neuroscience literature. Neuropsychologia, 53, 104-114.

Graesser, A. C., Singer, M., \& Trabasso, T. (1994). Constructing inferences during narrative text comprehension. Psychological Review, $101(3), 371$.

Griffiths, T. L., Steyvers, M., \& Tenenbaum, J. B. (2007). Topics in semantic representation. Psychological Review, 114(2), 211-244.

Hearnshaw, L. S. (1987). The shaping of modern psychology. Routledge \& Kegan Paul.

Howe, M. L., \& Wilkinson, S. (2011). Using story contexts to bias children's true and false memories. Journal of Experimental Child Psychology, 108(1), 77-95.

Ishwaran, H., \& James, L. F. (2001). Gibbs sampling methods for stick-breaking priors. Journal of the American Statistical Association, 96(453), 161-173.

Johansson, R., Oren, F., \& Holmqvist, K. (2018). Gaze patterns reveal how situation models and text representations contribute to episodic text memory. Cognition, 175, 53-68.

Jones, M., \& Love, B. C. (2011). Bayesian fundamentalism or enlightenment? On the explanatory status and theoretical contributions of bayesian models of cognition. Behavioral and Brain Sciences, 34(4), 169-188.

Kass, R. E., \& Raftery, A. E. (1995). Bayes factors. Journal of the American Statistical Association, 90(430), 773-795. 
Kersten, D., Mamassian, P., \& Yuille, A. (2004). Object Perception as Bayesian Inference. Annual Review of Psychology.

Kintsch, W. (1974). The representation of meaning in memory. Lawrence Erlbaum.

Kintsch, W. (1988). The role of knowledge in discourse comprehension - a construction integration model. Psychological Review, 95(2), 163-182.

Kintsch, W. (1998). Comprehension: A paradigm for cognition. Cambridge university press.

Kintsch, W., \& Van Dijk, T. A. (1978). Toward a model of text comprehension and production. Psychological Review, 85(5), 363.

Long, D. L., Prat, C., Johns, C., Morris, P., \& Jonathan, E. (2008). The importance of knowledge in vivid text memory: An individual-differences investigation of recollection and familiarity. Psychonomic Bulletin \& Review, 15(3), 604-609.

Mandler, J. M., \& Johnson, N. S. (1977). Remembrance of things parsed: Story structure and recall. Cognitive Psychology, 9(1), 111-151.

Marr, D. (1982). Vision. New York, NY: W. H. Freeman \& Company.

Mason, R. A., \& Just, M. A. (2004). How the brain processes causal inferences in text: A theoretical account of generation and integration component processes utilizing both cerebral hemispheres. Psychological Science, 15(1), 1-7.

McClelland, J. L., Botvinick, M. M., Noelle, D. C., Plaut, D. C., Rogers, T. T., Seidenberg, M. S., \& Smith, L. B. (2010). Letting structure emerge: Connectionist and dynamical systems approaches to cognition. Trends in Cognitive Sciences, 14(8), 348-356.

McElreath, R. (2015). Statistical rethinking: A Bayesian course with examples in $R$ and Stan. Chapman; Hall/CRC.

McKoon, G., \& Ratcliff, R. (1992). Inference during reading. Psychological Review, 99(3), 440.

McNamara, D. S., \& Kintsch, W. (1996). Learning from texts: Effects of prior knowledge and text coherence. Discourse Processes, 22(3), 247-288.

Minsky, M. (1975). A framework for representing knowledge. In P. H. Winston (Ed.), The psychology of computer vision (pp. 211-277). McGraw-Hill.

Neely, J. H. (1977). Semantic priming and retrieval from lexical memory: Roles of inhibitionless spreading activation and limited-capacity attention. Journal of Experimental Psychology: General, 106(3), 226.

Nelson, D. L., McEvoy, C. L., \& Schreiber, T. A. (2004). The university of south florida word association, rhyme and word fragment norms. Behavior Research Methods, Instruments, $E$ Computers, 36, 408-420.

Newman, D., Asuncion, A., Smyth, P., \& Welling, M. (2009). Distributed algorithms for topic models. The Journal of Machine Learning Research, 10, 1801-1828. 
Oaksford, M., \& Chater, N. (1994). A rational analysis of the selection task as optimal data selection. Psychological Review, $101(4), 608$.

Reyna, V. F., Corbin, J. C., Weldon, R. B., \& Brainerd, C. J. (2016). How fuzzy-trace theory predicts true and false memories for words, sentences, and narratives. Journal of Applied Research in Memory and Cognition, 5(1), 1-9.

Richmond, L. L., \& Zacks, J. M. (2017). Constructing experience: Event models from perception to action. Trends in Cognitive Sciences, 21(12), 962-980.

Roediger, H. L., \& McDermott, K. B. (1995). Creating false memories: Remembering words not presented in lists. Journal of Experimenal Psychology: Learning, Memory and Cognition, 21(4), 803-814.

Roediger, H. L., \& McDermott, K. B. (2000). Tricks of memory. Current Directions in Psychological Science, 9(4), 123-127.

Roediger, H. L., Watson, J. M., McDermott, K. B., \& Gallo, D. A. (2001). Factors that determine false recall: A multiple regression analysis. Psychonomic Bulletin 8 Review, 8(3), 385-407.

Rumelhart, D. E. (1975). Notes on a Schema for Stories. In A. Collins \& D. G. Bobrow (Eds.), Representation and understanding: Studies in cognitive science. Academic Press.

Rumelhart, D. E., \& McClelland, J. L. (1987). Parallel distributed processing: Explorations in the microstructure of cognition : Foundations, psychological and biological models. Mit Press.

Sanborn, A. N., Griffiths, T. L., \& Navarro, D. J. (2010). Rational approximations to rational models: Alternative algorithms for category learning. Psychological Review, $117(4), 1144$.

Schacter, D. L., Guerin, S. A., \& Jacques, P. L. S. (2011). Memory distortion: An adaptive perspective. Trends in Cognitive Sciences, 15(10), 467-474.

Schank, R. C., \& Abelson, R. P. (1977). Scripts, plans, goals, and understanding: An inquiry into human knowledge structures. Hillsdale, NJ: Lawrence Erlbaum Associates.

Schmalhofer, F., McDaniel, M. A., \& Keefe, D. (2002). A unified model for predictive and bridging inferences. Discourse Processes, 33(2), 105-132.

Schwartz, D. L., \& Bransford, J. D. (1998). A time for telling. Cognition and Instruction, $16(4), 475-5223$.

Shapiro, A. M. (2004). How including prior knowledge as a subject variable may change outcomes of learning research. American Educational Research Journal, 41(1), $159-189$.

Sorabji, R. (2004). Aristotle on memory. Bloomsbury Academic. 
Steyvers, M., \& Griffiths, T. (2007). Probabilisitic topic models. In T. K. Landauer, D. S. McNamara, S. Dennis, \& W. Kintsch (Eds.), Handbook of latent semantic analysis. Psychology Press.

Sulin, D., RA AND Dooling. (1974). Intrusion of a Thematic Idea in Retention Of Prose. Journal of Experimental Psychology, 103(2), 255-262.

Teh, Y. W., Jordan, M. I., Beal, M. J., \& Blei, D. M. (2004). Hierarchical Dirichlet Processes. In Advances in neural information processing systems (Vol. 17).

Teh, Y. W., Jordan, M. I., Beal, M. J., \& Blei, D. M. (2006). Hierarchical Dirichlet processes. Journal of the American Statistical Association, 101(476), 1566-1581.

Thorndyke, P. W. (1977). Cognitive structures in comprehension and memory of narrative discourse. Cognitive Psychology, 9(1), 77-110.

Thorndyke, P. W., \& Yekovich, F. R. (1980). A critique of schema-based theories of human story memory. Poetics, 9(1-3), 23-49.

Trabasso, T., \& Bartolone, J. (2003). Story understanding and counterfactual reasoning. Journal of Experimental Psychology: Learning, Memory, and Cognition, 29(5), 904.

Trabasso, T., \& Langston, M. (1998). Modeling causal integration and availability of information during comprehension of narrative texts. In H. Van Oostendorp \& S. R. Goldman (Eds.), The construction of mental representations during reading. Psychology Press.

Tzeng, Y., Van Den Broek, P., Kendeou, P., \& Lee, C. (2005). The computational implementation of the landscape model: Modeling inferential processes and memory representations of text comprehension. Behavior Research Methods, 37(2), 277-286.

Van den Broek, P. (2010). Using texts in science education: Cognitive processes and knowledge representation. Science, 328(5977), 453-456.

Van den Broek, P., Young, M., Tzeng, Y., Linderholm, T., \& others. (1998). The landscape model of reading: Inferences and the online construction of a memory representation. In H. Van Oostendorp \& S. R. Goldman (Eds.), The construction of mental representations during reading. Psychology Press.

Van Dijk, T. A., \& Kintsch, W. (1983). Strategies of discourse comprehension.

Van Oostendorp, H., \& Goldman, S. R. (1998). The construction of mental representations during reading. Psychology Press.

Xu, J., Kemeny, S., Park, G., Frattali, C., \& Braun, A. (2005). Language in context: Emergent features of word, sentence, and narrative comprehension. Neuroimage, $25(3), 1002-1015$.

Yang, X., Zhang, X., Yang, Y., \& Lin, N. (2018). How context features modulate the involvement of the working memory system during discourse comprehension. Neuropsychologia, 111, 36-44. 
Zwaan, R. A., \& Radvansky, G. A. (1998). Situation models in language comprehension and memory. Psychological Bulletin, 123(2), 162. 
Appendix A

Open, transparent, and reproducible research

All the code and data necessary to reproduce this work or to evaluate it in more detail has been made publicly available at Github (https://github.com/mark-andrews/ BayesianAccountMemoryText) and at Figshare (https://doi.org/10.6084/m9.figshare. 8246900). The code consists of R, RMarkdown, Python, Unix shell scripts, and Jupyter notebooks. All the data from behavioural experiments, as well as the data necessary for or created by the computational simulations are also included. There is approximately 20GB of files and data in total. Everything from the creation of the stimuli for the behavioural experiment, the sample size determination of the experiment, the creation of the BNC corpus for the topic modelling, the code the topic modelling, the computational modelling of the alternative (i.e., alternative to topic modelling) computational models, the post-processing of the computational models's data, the pre-processing of all the behavioural and other data prior to the final regression analyses, the final Bayesian regression analyses, as well as this manuscript with all the tables, figures etc are all reproducible using the scripts and data in this repository.

The sub-directories of this repository are as follows.

- data-analysis: R, Jags, Stan/brms code for Bayesian multilevel logistic regression.

- data-processing: Python code for pre-processing experimental data and preparing computational models' predictions for use in the regression analyses.

- make-corpus: Mostly Python code for the creation of the BNC corpus to be used in the probabilistic topic modelling.

- sample-size-determination: Mostly Python (calling $\mathrm{R}$ code) code for determining sample size for the behavioural experiment.

- stimuli-generation: Mostly Python code for generating the to-be-memorized texts and the memory word lists in the behavioural experiments.

- topic-modelling: Probabilistic topic modelling using the Gustav topic modelling toolbox.

- manuscript: The RMarkdown and $\mathrm{LAT}_{\mathrm{E}} \mathrm{X}$ manuscript, including $\mathrm{R}$ code for exploratory data analysis and visualizations. 


\author{
Appendix B \\ Sample size determination
}

We conducted numerical simulations to determine the total number of participants, the total number of different memory tests, and the number of memory tests per participant that were required to detect, with high probability, the predictive effect of the Bayesian (or alternative) computational model on recognition and recall memory scores.

Given that the outcome variables in the memory experiments are in the form of either memory recognition scores, which are binary values, or memory recall values, which are categorical (multinomial) values, and given that there is random inter-participant and inter-text variability in these outcome variables, the predictions of the Bayesian model and alternatives were tested using multilevel (i.e., random slopes and intercepts) logistic regression analyses with one predictor variable that gives the predictions of the Bayesian (or alternative) computational model. First, we generated data from a multilevel logistic regression model under the following conditions:

- Total number of participants ranging from 25 to 150.

- Total number of distinct memory tests ranging from 5 to 50 .

- Total number of tests per subject ranging from 3 to 5 .

- Effect size of the predictor ranging from low to high on a standard scale.

- Inter-subject variation, and inter-text variation, ranging from low to high.

We generated 50,000 data sets for each combination of these settings ${ }^{8}$, and calculated the probability of obtaining Bayesian Information Criterion (BIC) scores for the inferred multilevel logistic regression that was less than 10 units below that of a null model. The null model was identical in all respects to the multilevel logistic regression, but with the coefficient for the predictor variable being set to zero. These simulations made clear that if the experiment uses a large number of participants (i.e. at least 150), and a large number of texts (i.e. at least 50), then there is a high probability of detecting the predictive effect of the computational model. This is the case even if we assume that the true effect size of the predictive effect is low on a standard scale. In addition, we have high probability of detecting the effect even if each participant performs only three memory tests each.

Additional analyses confirmed that if we had 10 tests in total, and each participant performed each one of these tests, then even with large numbers of participants, there is high probability of detecting effects only if the effects are assumed to be large or very large on a standard scale. This is an important finding given that experimental designs where all participants are exposed to all the stimuli are very common designs. Our simulations show, however, that these are sub-optimal designs, and that using a large number of tests, but with each participant performing only a small sample of these tests, are to be preferred.

How to obtain further details about these simulations, including all the $\mathrm{R}$ and Python code used to perform them are provided in Appendix A.

\footnotetext{
${ }^{8}$ These simulations were run by parallel processes and took approximately 60 hours on a 16 core Xeon E5-2690.
} 


\section{Appendix C \\ Measuring out-of-sample predictive performance}

In order to understand how we calculate our models' out-of-sample predictive performance, we must first provide some background. Any statistical model's out-of-sample predictive performance measures how well it can predict future, or alternative, data sets that are generated by the same underlying process as the data to which it is fitted. In more detail, in general, any data set $\mathcal{D}$ can be described as a sample from an unknown true statistical model $f_{\text {True }}$. A future or alternative data point that is also generated by $f_{\text {True }}$ may be denoted $\tilde{y}$. How well any hypothetical statistical model, denoted $f_{\mathrm{H}}$, that has been fitted to $\mathcal{D}$ can predict $\tilde{y}$ is given by its posterior predictive density, or equivalently the logarithm thereof:

$$
\log \mathrm{P}(\tilde{y} \mid \mathcal{D}, \mathrm{H})=\log \int \mathrm{P}(\tilde{y} \mid \theta) f_{\mathrm{H}}(\theta \mid \mathcal{D}) d \theta,
$$

where $f_{\mathrm{H}}(\theta \mid \mathcal{D})$ is the posterior distribution over all the $f_{\mathrm{H}}$ model's unknown variables, which are generically denoted by $\theta$. The average out-of-sample predictive performance of model $f_{\mathrm{H}}$, again when fitted to $\mathcal{D}$, is its log posterior prediction density of a future data point averaged over all possible such data points, i.e,

$$
\langle\log \mathrm{P}(\tilde{y} \mid \mathcal{D}, \mathrm{H})\rangle_{f_{\text {True }}}=\int \log \mathrm{P}(\tilde{y} \mid \mathcal{D}, \mathrm{H}) f_{\text {True }}(\tilde{y}) d \tilde{y} .
$$

When we have $n$ separate future, or alternative, data points $\tilde{y}_{1}, \tilde{y}_{2} \ldots \tilde{y}_{n}$, how well model $f_{\text {True }}$ can predict this entire data set is referred to as the expected log pointwise predictive density (elppd) for the new data data-set (see Gelman et al., 2014, p. 168), and is defined as

$$
\operatorname{elppd}=\sum_{i=1}^{n}\left\langle\log \mathrm{P}\left(\tilde{y}_{i} \mid \mathcal{D}, \mathrm{H}\right)\right\rangle_{f_{\text {True }}} .
$$

Clearly, given that we do not know $f_{\text {True }}$, we do not and can not know the values of all possible future data-sets, and so can not compute the elppd for our model. However, various approximations to elppd are possible (see Gelman et al., 2014, Chapter 7, for discussion). These include cross-validation, and particularly leave-one-out cross-validation, Akaike Information Criterion (AIC), Deviance Information Criterion (DIC), and Watanabe Akaike Information Criterion (WAIC). Here, we will use WAIC. It has been shown to be more generally or widely applicable than either AIC or DIC, and is a close approximation to leave-one-out cross-validation, yet can be calculated easily from a model's posterior samples (Watanabe, 2010). WAIC is calculated as -2 times the following quantity:

$$
\sum_{i=1}^{n} \log \left(\frac{1}{S} \sum_{s=1}^{S} \mathrm{P}\left(y_{i} \mid \theta^{s}\right)\right)-\sum_{i=1}^{n} V_{s=1}^{S}\left(\log \mathrm{P}\left(y_{i} \mid \theta^{s}\right)\right),
$$

where $y_{i}$ is the $i$ th observed variable, $\theta^{s}$ is a single sample from the posterior over all the regression model's unknown variables (i.e. we use $\theta$ to generically denote all unknown variables in the model), and we assume we have $S$ samples from the posterior distribution over $\theta$. The term $V_{s=1}^{S}(\cdot)$ signifies the variance of its arguments.

Gelman, A., Carlin, J. B., Stern, H. S., Dunson, D. B., Vehtari, A., \& Rubin, D. B. (2014). Bayesian data analysis (3rd ed.). New York, NY: Taylor \& Francis. 
Watanabe, S. (2010). Asymptotic equivalence of Bayes cross validation and widely applicable information criterion in singular learning theory. Journal of Machine Learning Research, 11 (Dec), 3571-3594. 